\title{
Unshared Secret Key Cryptography
}

\author{
Shuiyin Liu, Member, IEEE, Yi Hong, Senior Member, IEEE, and Emanuele Viterbo, Fellow, IEEE
}

\begin{abstract}
Current security techniques can be implemented with either secret key exchange or physical-layer wiretap codes. In this paper, we investigate an alternative solution for MIMO wiretap channels. Inspired by the artificial noise (AN) technique, we propose the unshared secret key (USK) cryptosystem, where the $\mathrm{AN}$ is redesigned as a one-time pad secret key aligned within the null space between a transmitter and a legitimate receiver. The proposed USK cryptosystem is a new physical-layer cryptographic scheme, which was obtained by combining traditional network-layer cryptography and physical-layer security. Unlike previously studied AN techniques, rather than ensuring nonzero secrecy capacity, the USK is valid for an infinite lattice input alphabet and guarantees Shannon's ideal secrecy and perfect secrecy without the need for secret key exchange. We then show how ideal secrecy can be obtained for finite lattice constellations with an arbitrarily small outage.
\end{abstract}

Index Terms-Perfect secrecy, ideal secrecy, secret key, physical layer security, MIMO wiretap channel.

\section{INTRODUCTION}

$\mathbf{T}$ HE broadcast characteristics of wireless communication systems are struggling to provide security and privacy. Research on secure communication falls into two categories: network layer cryptography and physical layer security. The former assumes that the physical layer provides error-free data links, in which security depends on encryption. In the latter, the strategy is to use the characteristics of wireless channels to protect the secret data from eavesdropping without the need of encryption. Despite the differences between these categories, both are rooted in Shannon's perfect secrecy [1], which is defined as the mutual information $I(\mathbf{u} ; \mathbf{y})=0$; that is, the secret message $\mathbf{u}$ and the eavesdropper's received message $\mathbf{y}$ are mutually independent. Perfect secrecy requires one-time pad secret key $\mathbf{v}$ [1]. A weaker version of perfect secrecy is ideal secrecy [1], in which no matter how much of $\mathbf{y}$ is intercepted, there is no unique solution of $\mathbf{u}$ and $\mathbf{v}$ but many solutions of comparable probability.

Manuscript received November 4, 2013; revised April 17, 2014 and August 5, 2014; accepted October 13, 2014. Date of publication October 20, 2014; date of current version December 8, 2014. This work was performed at the Monash Software Defined Telecommunications Laboratory. This work was supported in part by the Monash Professorial Fellowship, by the 2013 Monash Faculty of Engineering Seed Funding Scheme, and by the Australian Research Council Discovery Project under Grant ARC DP130100336. The associate editor coordinating the review of this paper and approving it for publication was J. Wu.

The authors are with the Department of Electrical and Computer Systems Engineering, Faculty of Engineering, Monash University, Melbourne, VIC 3800 Australia (e-mail: shuiyin.liu@monash.edu; yi.hong@ monash.edu; emanuele.viterbo@monash.edu).

Color versions of one or more of the figures in this paper are available online at http://ieeexplore.iee.org.

Digital Object Identifier 10.1109/TWC.2014.2364022
Wyner [2] introduced physical layer security by replacing the shared secret key in Shannon's model with channel noise, achieving weak secrecy $\left(\lim _{n \rightarrow \infty} \frac{1}{n} I(\mathbf{u} ; \mathbf{y})=0\right)$ through channel coding as the codeword length $n$ goes to infinity. Csiszár subsequently proposed strong secrecy [3] based upon $\lim _{n \rightarrow \infty} I(\mathbf{u} ; \mathbf{y})=$ 0 , which further reduced information leakage. These pioneering results require that the intended receiver has a better channel than the eavesdropper, leading to a long line of research that relies on noise or fading to degrade the eavesdropper's channel. Here, the secrecy capacity is defined as a measure of the transmission rate, below which the eavesdropper can recover no information [4]. For Gaussian wiretap channels with a helping interferer, Tang et al. [5] studied achievable secrecy rate and secrecy capacity. For wireless fading channels and multipleinput multiple-output (MIMO) wiretap channels, Gopala et al. [6] and Liu et al. [7] derived secrecy capacities. In the context of wiretap code design, polar codes achieving strong secrecy over discrete memoryless channels have been proposed in [8]. For Gaussian wiretap channels, nested lattice codes achieving strong secrecy were proposed in [9]. The impact of finite code length and finite constellations on Eve's equivocation rate was studied in [10]. Physical layer security schemes, in general, require an infinite-length wiretap code to approach the secrecy capacity; this limits the applicability of these schemes to practical communication systems.

In contrast to physical layer security, traditional cryptographic techniques can protect the secret message, even when secrecy capacity is zero. Its aim is to achieve semantic secrecy [11], so that it is physically infeasible to extract any information about $\mathbf{u}$ due to the very high computational complexity involved. The most widely used cryptographic technology is public-key cryptography [12], which requires two separate keys: a public key that encrypts the plaintext and a secret key that decrypts the ciphertext. An example is the NTRU cryptosystem [13], where the secret key is based on a short vector of a convolutional modular lattice, $\Lambda$, and the public key corresponds to the Hermite normal form basis of $\Lambda$ [14]. For wiretap channels, public-key cryptography has been extensively studied in [15], [16], which focus on issues of secretkey generation and distribution problems. Bloch et al. [17] showed how to implement secret-key agreement using lowdensity parity-check (LDPC) codes. In [18], turbo codes are introduced to speed up the encryption and decryption processes of the advanced encryption standard (AES) cryptosystems. Although traditional cryptographic techniques can be applied independently to communication channels, the exchange of secret keys between transmitter and intended receiver is required. A significant challenge is to reduce the risk of key disclosure during its distribution. 
Despite the similarities between cryptography and physical layer security, and the potential for major advances in cryptography through combining their advantages, the theoretical connections between them have not yet been investigated. One direction has been to add controlled interference at the eavesdropper side - that is, to jam the eavesdropper at the physical layer. This idea extends previous studies that were limited to the assumptions on the eavesdropper's channel noise. In the literature, it is commonly assumed that the transmitted message and jamming signal follow a multivariate Gaussian distribution. The standard strategy of existing jamming techniques, such as artificial noise (AN) [19] and the cooperative jammer [20], is to ensure theoretical non-zero secrecy capacity. In [21], we proposed a variant of AN using a finite $M$-QAM alphabet, called practical secrecy (PS) scheme, where, instead of increasing the secrecy rate with AN, the eavesdropper's error probability is maximized.

In this work, we analyze the security of the PS scheme from an information theoretical perspective. This theoretical advance shows that the PS scheme is de facto an unshared secret key (USK) cryptosystem, where AN serves as an unshared one-time pad secret key. The result is a development of our understanding of the benefits of AN, with a cryptographic perspective. We show that the USK provides Shannon's ideal secrecy, with no secret key exchange, under Goel et al.'s assumptions on the physical channels that enable the use of the AN scheme.

Our work differs from previous studies of AN [19], [22], because it puts forward four new aspects that were not previously accounted for:

1) Shannon's secrecy: we aim at achieving Shannon's ideal secrecy and perfect secrecy, rather than ensuring nonzero secrecy capacity. We show that perfect secrecy is achieved in the high-power AN limit.

2) Finite alphabet based on QAM signaling: with practical perspective, we use finite input alphabets rather than the Gaussian input.

3) Artificial noise: we have no special requirement of the distribution of AN; that is, not necessarily Gaussian.

4) Secrecy outage: we show that Shannon's ideal secrecy can be achieved for finite signal constellations with an arbitrarily small outage probability.

Section II presents the system model. Sections III and IV describe the USK cryptosystem with infinite lattice constellations. Sections V and VI analyze the USK cryptosystem with finite lattice constellations. Section VII provides a discussion on open questions. Section VIII sets out the theoretical and applied conclusions. The Appendix contains the proofs of the theorems.

Notation: Matrices and column vectors are denoted by upper and lowercase boldface letters, and the Hermitian transpose, inverse, pseudo-inverse of a matrix $\mathbf{B}$ by $\mathbf{B}^{H}, \mathbf{B}^{-1}$, and $\mathbf{B}^{\dagger}$, respectively. The inner product in the Euclidean space between vectors $\mathbf{u}$ and $\mathbf{v}$ is defined as $\langle\mathbf{u}, \mathbf{v}\rangle=\mathbf{u}^{T} \mathbf{v}$, and the Euclidean length $\|\mathbf{u}\|=\sqrt{\langle\mathbf{u}, \mathbf{u}\rangle}$. The Frobenius norm of matrix $\mathbf{A}$ is denoted by $\|\mathbf{A}\|_{F}$. Let $\left\{X_{n}, X\right\}$ be defined on the same probability space. We write $X_{n} \stackrel{\text { a.s. }}{\rightarrow} X$ if $X_{n}$ converges to $X$ almost surely or with probability one.
We use the standard asymptotic notation $f(x)=O(g(x))$ when $\lim \sup _{x \rightarrow \infty}|f(x) / g(x)|<\infty . \mathbf{0}_{m \times n}$ denotes an $m \times n$ null matrix. $\mathbf{I}_{n}$ denotes the identity matrix of size $n$. We write $\triangleq$ for equality in definition. $\operatorname{vol}(S)$ denotes the Euclidean volume of $S$. The cardinality of a set $A$ is defined as $|A|$.

A circularly symmetric complex Gaussian random variable $x$ with variance $\sigma^{2}$ is defined as $x \sim \mathcal{N}_{\mathbb{C}}\left(0, \sigma^{2}\right)$. A Chi-squared distributed random variable $x$ with $k$ degrees of freedom is defined as $x \backsim \mathcal{X}^{2}(k)$. The gamma function is represented by $\Gamma(x)$. The real, complex, integer and complex integer numbers are denoted by $\mathbb{R}, \mathbb{C}, \mathbb{Z}$, and $\mathbb{Z}[i]$, respectively. $\mathrm{E}(x)$ and $\operatorname{Var}(x)$ represent the mean and variance of the random variable $x$. $\Re(\cdot)$ and $\Im(\cdot)$ represent real and imaginary parts of a complex number. $H(\cdot), H(\cdot \mid \cdot)$ and $I(\cdot)$ represent entropy, conditional entropy and mutual information, respectively.

\section{System Model}

The MIMO wiretap system model is given as follows. The number of antennas at the transmitter (Alice), the intended receiver (Bob), and the passive eavesdropper (Eve) are denoted by $N_{\mathrm{A}}, N_{\mathrm{B}}$, and $N_{\mathrm{E}}$, respectively. Alice would like to communicate with Bob with arbitrarily low probability of error, while maintaining privacy and confidentiality. Alice transmits the information signal $\mathbf{x}$, and Bob and Eve receive $\mathbf{z}$ and $\mathbf{y}$, respectively, given by

$$
\begin{aligned}
& \mathbf{z}=\mathbf{H} \mathbf{x}+\mathbf{n}_{\mathrm{B}}, \\
& \mathbf{y}=\mathbf{G} \mathbf{x}+\mathbf{n}_{\mathrm{E}},
\end{aligned}
$$

where $\mathbf{H} \in \mathbb{C}^{N_{\mathrm{B}} \times N_{\mathrm{A}}}$ and $\mathbf{G} \in \mathbb{C}^{N_{\mathrm{E}} \times N_{\mathrm{A}}}$ are the channel matrices of Bob and Eve. We assume that all the channel matrix elements are i.i.d. $\mathcal{N}_{\mathbb{C}}(0,1)$ random variables (i.e., Bob and Eve are not co-located). We assume that the noise vectors $\mathbf{n}_{\mathrm{B}}$ and $\mathbf{n}_{\mathrm{E}}$ have i.i.d. $\mathcal{N}_{\mathbb{C}}\left(0, \sigma_{\mathrm{B}}^{2}\right)$ and $\mathcal{N}_{\mathbb{C}}\left(0, \sigma_{\mathrm{E}}^{2}\right)$ components, respectively.

In this work, we assume that

1) Alice knows the realization of $\mathbf{H}$.

2) Alice only knows the statistics of $\mathbf{G}$, which varies in each transmission.

3) Eve knows the realizations of $\mathbf{H}$ and $\mathbf{G}$.

No assumption is needed about the statistics of $\mathbf{H}$ during transmission, since its realization is known to Alice and Eve.

Our secure transmission strategy is based on the artificial noise scheme [19] and the practical secrecy scheme [21], which are summarized below.

\section{A. Artificial Noise Scheme}

In the AN scheme [19], $N_{\mathrm{B}}$ is assumed to be smaller than $N_{\mathrm{A}}$, thus $\mathbf{H}$ has a non-trivial null space with an orthonormal basis given by columns of the matrix $\mathbf{Z}=\operatorname{null}(\mathbf{H}) \in$ $\mathbb{C}^{N_{\mathrm{A}} \times\left(N_{\mathrm{A}}-N_{\mathrm{B}}\right)}$, i.e.,

$$
\mathbf{H Z}=\mathbf{0}_{N_{\mathrm{B}} \times N_{\mathrm{B}}} .
$$

Let $\mathbf{u} \in \mathbb{C}^{N_{\mathrm{B}} \times 1}$ be the transmitted vector carrying the information, and let $\mathbf{v} \in \mathbb{C}^{\left(N_{\mathrm{A}}-N_{\mathrm{B}}\right) \times 1}$ represent the "artificial noise" generated by Alice but is unknown to Bob and Eve. 
Alice performs SVD precoding and transmits

$$
\mathbf{x}=\mathbf{V}\left[\begin{array}{l}
\mathbf{u} \\
\mathbf{v}
\end{array}\right]=\mathbf{V}_{1} \mathbf{u}+\mathbf{Z} \mathbf{v}
$$

where the columns of $\mathbf{V}=\left[\mathbf{V}_{1}, \mathbf{Z}\right]$ are the right-singular vectors of $\mathbf{H}$ (i.e., $\mathbf{H}=\mathbf{U} \boldsymbol{\Lambda} \mathbf{V}^{H}$, where $\mathbf{U} \in \mathbb{C}^{N_{\mathrm{B}} \times N_{\mathrm{B}}}, \boldsymbol{\Lambda} \in$ $\left.\mathbb{C}^{N_{\mathrm{B}} \times N_{\mathrm{A}}}, \mathbf{V} \in \mathbb{C}^{N_{\mathrm{A}} \times N_{\mathrm{A}}}, \mathbf{U}^{H} \mathbf{U}=\mathbf{I}_{N_{\mathrm{B}}}, \mathbf{V}^{H} \mathbf{V}=\mathbf{I}_{N_{\mathrm{A}}}\right)$.

Due to the orthogonality between $\mathbf{V}_{1}$ and $\mathbf{Z}$, the total transmission power $\|\mathbf{x}\|^{2}$ can be written as

$$
\|\mathbf{x}\|^{2}=\|\mathbf{u}\|^{2}+\|\mathbf{v}\|^{2} .
$$

Alice has an average transmit power constraint $P$,

$$
P \geq \mathrm{E}\left(\|\mathbf{x}\|^{2}\right)=\mathrm{E}\left(\|\mathbf{u}\|^{2}\right)+\mathrm{E}\left(\|\mathbf{v}\|^{2}\right) .
$$

The AN scheme in [19] is based on the assumptions below:

1) $\mathbf{u}$ and $\mathbf{v}$ are assumed to be Gaussian random vectors.

2) $N_{\mathrm{A}}>N_{\mathrm{B}}, N_{\mathrm{A}}>N_{\mathrm{E}}$ and $N_{\mathrm{E}} \geq N_{\mathrm{B}}$.

The condition $N_{\mathrm{E}} \geq N_{\mathrm{B}}$ guarantees that Eve has at least the same number of degree of freedom as Bob. This puts Eve in the position of not losing a-priori any information that Bob could receive.

Equations (1) and (2) can then be rewritten as

$$
\begin{aligned}
& \mathbf{z}=\mathbf{H V}_{1} \mathbf{u}+\mathbf{n}_{\mathrm{B}}, \\
& \mathbf{y}=\mathbf{G V}_{1} \mathbf{u}+\mathbf{G Z} \mathbf{v}+\mathbf{n}_{\mathrm{E}}
\end{aligned}
$$

and show that $\mathbf{v}$ only degrades Eve's reception, but not Bob's.

The purpose of the AN scheme is to degrade Eve's channel, so that the secrecy capacity is positive [19]. Like other wiretap schemes, to achieve the secrecy capacity, explicit wiretap codes are required. A strong secrecy rate $R$ is achievable if there exist a sequence of wiretap codes $\left\{\mathcal{C}_{n}\right\}$ of increasing length $n$ and rate $R$, such that both Bob's error probability and the amount of information obtained by Eve approach zero when $n \rightarrow \infty$ [3], [9], i.e.,

$$
\begin{array}{lr}
\lim _{n \rightarrow \infty} \operatorname{Pr}\{\hat{\mathbf{u}} \neq \mathbf{u}\}=0, & \text { (reliability) } \\
\lim _{n \rightarrow \infty} I(\mathbf{u} ; \mathbf{y})=0, & \text { (strong secrecy) }
\end{array}
$$

where $\hat{\mathbf{u}}$ represents Bob's estimation of $\mathbf{u}$.

\section{B. Practical Secrecy Scheme}

Rather than attempting to increase secrecy rate, in [21], we proposed a variant of the AN scheme, named practical secrecy (PS) scheme, where Eve's error probability is maximized. Although the transmission model is the same as that of $\mathrm{AN}$, the most important difference lies in the distributions of $\mathbf{u}$ and $\mathbf{v}$ :

1) $M$-QAM transmitted symbols: $\mathbf{u} \in \mathcal{Q}^{N_{\mathrm{B}}}$ with uniform distribution, where $\Re(\mathcal{Q})=\Im(\mathcal{Q})=\{-\sqrt{M}+1$, $-\sqrt{M}+3, \ldots, \sqrt{M}-1\}$.

2) There is no requirement on the distribution of $\mathbf{v}$.

Different from the AN scheme, where the achievability of security is based on an infinite-length wiretap code, the PS scheme [21] is designed for practical communication systems, that make use of finite input alphabets based on $M$-QAM transmitted symbols. The aim is to ensure that Eve's block error probability approaches one with minimum distance decoding, (e.g., sphere decoder), rather than strong secrecy. However, this security criterion is not satisfactory from an informationtheoretic security viewpoint, as it may not ensure security for all information bits within a message.

\section{Proposed AN Scheme}

Different from the original AN scheme [19], in this work, we set a peak AN power constraint,

$$
P_{\mathrm{v}} \geq\|\mathbf{v}\|^{2} .
$$

This peak power constraint is essential to prove the secrecy of USK, as detailed in Section III-A.

Moreover, we consider two lattice constellation models:

1) Infinite constellations with average power constraint

2) Finite constellations with peak power constraint

We focus on information theoretic security, hence, our analysis will focus on Eve's equivocation $H(\mathbf{u} \mid \mathbf{y})$.

Throughout the paper, we consider the worst-case scenario (for Alice) that Eve's channel is noiseless, i.e.,

$$
\mathbf{y}=\mathbf{G V}_{1} \mathbf{u}+\mathbf{G Z \mathbf { v }}
$$

Using Data Processing Inequality, it is simple to show that Eve's channel noise can only increase her equivocation:

$$
H\left(\mathbf{u} \mid \mathbf{G V}_{1} \mathbf{u}+\mathbf{G} \mathbf{Z} \mathbf{v}\right) \leq H\left(\mathbf{u} \mid \mathbf{G V}_{1} \mathbf{u}+\mathbf{G} \mathbf{Z} \mathbf{v}+\mathbf{n}_{\mathrm{E}}\right) .
$$

We further consider the worst-case scenario (for Alice) that Eve's antenna array elements are uncorrelated, i.e., the columns of $\mathbf{G}$ are zero-mean independent complex Gaussian vectors with an identity covariance matrix.

For a general complex Gaussian random matrix $\hat{\mathbf{G}}$ with an arbitrary non-singular covariance matrix $\Sigma$ (which is the covariance matrix of Eve's antenna array), we can write

$$
\hat{\mathbf{G}}=\Sigma^{1 / 2} \mathbf{G} \text {. }
$$

Using Data Processing Inequality, it is simple to show that Eve's antenna correlation can only increase her equivocation:

$$
H\left(\mathbf{u} \mid \mathbf{G} \mathbf{V}_{1} \mathbf{u}+\mathbf{G} \mathbf{Z} \mathbf{v}\right) \leq H\left(\mathbf{u} \mid \Sigma^{1 / 2} \mathbf{G V}_{1} \mathbf{u}+\Sigma^{1 / 2} \mathbf{G} \mathbf{Z v}\right) .
$$

Remark 1: Throughout this paper, the proposed security analysis of USK scheme is valid for a complex Gaussian random matrix $\mathbf{G}$ with an arbitrary non-singular covariance matrix $\Sigma$. The extension to USK of other distributed random matrix $\mathbf{G}$ will be studied in our future work.

\section{Shannon's Secrecy}

We consider a cryptosystem where a sequence of $K$ messages $\left\{\mathbf{m}_{i}\right\}_{1}^{K}$ are enciphered into the cryptograms $\left\{\mathbf{y}_{i}\right\}_{1}^{K}$ using a sequence of secret keys $\left\{k_{i}\right\}_{1}^{K}$. We recall from [1] the definition of Shannon's ideal secrecy and perfect secrecy. 
Definition 1: A secrecy system is ideal when

$$
\begin{gathered}
\lim _{K \rightarrow \infty} H\left(\left\{\mathbf{m}_{i}\right\}_{1}^{K} \mid\left\{\mathbf{y}_{i}\right\}_{1}^{K}\right) \neq 0, \\
\lim _{K \rightarrow \infty} H\left(\left\{k_{i}\right\}_{1}^{K} \mid\left\{\mathbf{y}_{i}\right\}_{1}^{K}\right) \neq 0 .
\end{gathered}
$$

Shannon explained the concept of ideal secrecy in [1] as: "No matter how much material is intercepted, there is not a unique solution but many of comparable probability." It was discussed in [23] how a system achieving ideal secrecy is indeed unbreakable.

Definition 2: A secrecy system is perfect when

$$
H\left(\left\{\mathbf{m}_{i}\right\}_{1}^{K} \mid\left\{\mathbf{y}_{i}\right\}_{1}^{K}\right)=H\left(\left\{\mathbf{m}_{i}\right\}_{1}^{K}\right) .
$$

In the special case that $\left\{\mathbf{m}_{i}\right\}_{1}^{K}$ and $\left\{k_{i}\right\}_{1}^{K}$ are mutually independent, using the entropy chain rule, we have

$$
\begin{gathered}
H\left(\left\{\mathbf{m}_{i}\right\}_{1}^{K}\right)=\sum_{i=1}^{K} H\left(\mathbf{m}_{i}\right), \\
H\left(\left\{\mathbf{m}_{i}\right\}_{1}^{K} \mid\left\{\mathbf{y}_{i}\right\}_{1}^{K}\right)=\sum_{i=1}^{K} H\left(\mathbf{m}_{i} \mid \mathbf{y}_{i}\right), \\
H\left(\left\{k_{i}\right\}_{1}^{K} \mid\left\{\mathbf{y}_{i}\right\}_{1}^{K}\right)=\sum_{i=1}^{K} H\left(k_{i} \mid \mathbf{y}_{i}\right) .
\end{gathered}
$$

From (17) and (18), ideal secrecy is achieved if $H\left(\mathbf{m}_{i} \mid \mathbf{y}_{i}\right) \neq$ 0 and $H\left(k_{i} \mid \mathbf{y}_{i}\right) \neq 0$ for one of any $i$. To protect all the messages, in this work, we use a slightly stronger condition as our design criterion for ideal secrecy, given by

Definition 3: If $\left\{\mathbf{m}_{i}\right\}_{1}^{K}$ and $\left\{k_{i}\right\}_{1}^{K}$ are mutually independent, a secrecy system is ideal when

$$
H\left(\mathbf{m}_{i} \mid \mathbf{y}_{i}\right) \neq 0 \text { and } H\left(k_{i} \mid \mathbf{y}_{i}\right) \neq 0, \quad \text { for all } i .
$$

From (16) and (17), perfect secrecy is achieved when

$$
H\left(\mathbf{m}_{i} \mid \mathbf{y}_{i}\right)=H\left(\mathbf{m}_{i}\right), \quad \text { for all } i .
$$

An overview of measures on information-theoretic security can be found in [24].

\section{E. Lattice Preliminaries}

To describe our scheme, it is convenient to introduce some lattice preliminaries. An $n$-dimensional complex lattice $\Lambda_{\mathbb{C}}$ in a complex space $\mathbb{C}^{m}(n \leq m)$ is the discrete set defined by:

$$
\Lambda_{\mathbb{C}}=\left\{\mathbf{B u}: \mathbf{u} \in \mathbb{Z}[i]^{n}\right\},
$$

where the basis matrix $\mathbf{B}=\left[\mathbf{b}_{1} \cdots \mathbf{b}_{n}\right]$ has linearly independent columns.

$\Lambda_{\mathbb{C}}$ can also be easily represented as $2 n$-dimensional real lattice $\Lambda_{\mathbb{R}}$ [25]. In what follows, we introduce some lattice parameters of $\Lambda_{\mathbb{C}}$, which have a corresponding value for $\Lambda_{\mathbb{R}}$. The Voronoi region of $\Lambda_{\mathbb{C}}$, defined by

$$
\mathcal{V}_{i}\left(\Lambda_{\mathbb{C}}\right)=\left\{\mathbf{y} \in \mathbb{C}^{m}:\left\|\mathbf{y}-\mathbf{x}_{i}\right\| \leq\left\|\mathbf{y}-\mathbf{x}_{j}\right\|, \forall \mathbf{x}_{i} \neq \mathbf{x}_{j}\right\},
$$

gives the nearest neighbor decoding region of lattice point $\mathbf{x}_{i}$.
The volume of any $\mathcal{V}_{i}\left(\Lambda_{\mathbb{C}}\right)$, defined as $\operatorname{vol}\left(\Lambda_{\mathbb{C}}\right) \triangleq\left|\operatorname{det}\left(\mathbf{B}^{H} \mathbf{B}\right)\right|$, is equivalent to the volume of the corresponding real lattice.

The effective radius of $\Lambda_{\mathbb{C}}$, denoted by $r_{\text {eff }}\left(\Lambda_{\mathbb{C}}\right)$, is the radius of a sphere of volume $\operatorname{vol}\left(\Lambda_{\mathbb{C}}\right)$ [26]. For large $n$, it is approximately

$$
r_{\mathrm{eff}}\left(\Lambda_{\mathbb{C}}\right) \approx \sqrt{n /(\pi e)} \operatorname{vol}\left(\Lambda_{\mathbb{C}}\right)^{\frac{1}{2 n}} .
$$

\section{Unshared SECRET Key CRyptosystem WiTH INFINITE CONSTELLATIONS}

In this section, we consider the system model with an infinite lattice constellations, satisfying the average transmit power constraint. This provides the theoretical basis for unshared secret key cryptosystems.

\section{A. Encryption}

We consider a sequence of $K$ mutually independent messages $\left\{\mathbf{m}_{i}\right\}_{1}^{K}$, where each one is mapped to a transmitted vector $\mathbf{u} \in \mathbb{Z}[i]^{N_{\mathrm{B}}}$. The probability distribution of $\mathbf{u}$ can be arbitrary, but has finite $\mathrm{E}\left(\|\mathbf{u}\|^{2}\right)$. To secure the $K$ transmitted vectors $\left\{\mathbf{u}_{i}\right\}_{1}^{K}$, Alice enciphers $\left\{\mathbf{u}_{i}\right\}_{1}^{K}$ into the cryptograms $\left\{\mathbf{y}_{i}\right\}_{1}^{K}$ using a sequence of mutually independent secret keys $\left\{\mathbf{v}_{i}\right\}_{1}^{K}$. We assume that $\left\{\mathbf{v}_{i}\right\}_{1}^{K}$ and $\left\{\mathbf{u}_{i}\right\}_{1}^{K}$ are mutually independent, and $\left\{\mathbf{G}_{i}\right\}_{1}^{K}$ are mutually independent Gaussian random matrices. No assumption is needed about the statistics of $\left\{\mathbf{H}_{i}\right\}_{1}^{K}$ across the $K$ channel uses, since its realization is known to both Alice and Eve.

Since $\left\{\mathbf{v}_{i}\right\}_{1}^{K}$ and $\left\{\mathbf{u}_{i}\right\}_{1}^{K}$ are mutually independent, from (19) and (20), we only need to demonstrate the encryption process for one transmitted vector $\mathbf{u}_{i}$. For simplicity, we drop the subscript $i$.

For each $\mathbf{u}$, Alice randomly and independently (without any predefined distribution) chooses a one-time pad secret key $\mathbf{v}$, from a ball of radius $\sqrt{\overline{P_{\mathrm{v}}}}$ :

$$
S \triangleq\left\{\mathbf{v} \in \mathbb{C}^{N_{\mathrm{A}}-N_{\mathrm{B}}}:\|\mathbf{v}\|^{2} \leq P_{\mathrm{v}}\right\},
$$

and transmits

$$
\mathbf{x}=\mathbf{V}_{1} \mathbf{u}+\mathbf{Z} \mathbf{v}
$$

In the worst-case scenario, when $\mathbf{n}_{\mathrm{E}}=\mathbf{0}$, Eve will receive (10), i.e.,

$$
\mathbf{y}=\mathbf{G} \mathbf{V}_{1} \mathbf{u}+\tilde{\mathbf{n}}_{\mathrm{v}}
$$

where $\tilde{\mathbf{n}}_{\mathrm{v}}=\mathbf{G Z \mathbf { v }}$.

The signal model (24) can be interpreted as an encryption algorithm, that is, the secret message $\mathbf{u}$ is encrypted to $\mathbf{y}$ using a secret key $\mathbf{v}$, which is not released neither to Bob nor to Eve.

The message $\mathbf{u}$ is received by Eve as a lattice point (see Fig. 1) in:

$$
\Lambda_{\mathbb{C}}=\left\{\mathbf{G V}_{1} \mathbf{u}, \mathbf{u} \in \mathbb{Z}[i]^{N_{\mathrm{B}}}\right\}
$$




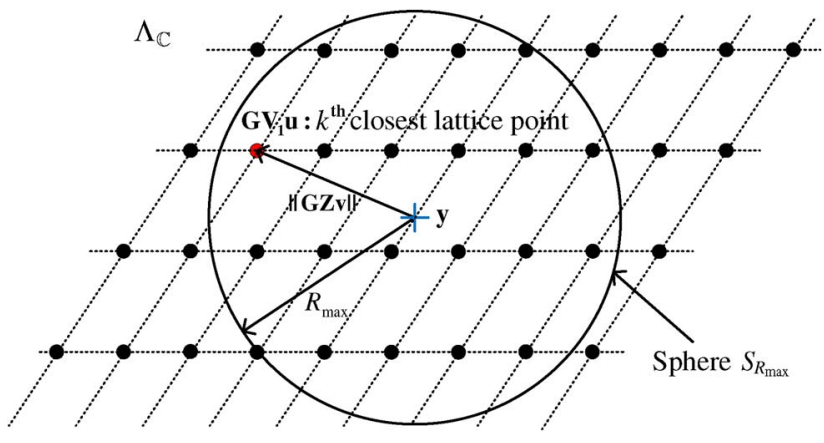

Fig. 1. The USK cryptosystem with infinite constellations.

This enables us to partition the set $S$ into $D$ disjoint subsets $S_{1}, \ldots, S_{D}$, such that

$$
S=\bigcup_{k=1}^{D} S_{k}
$$

where

$S_{k} \triangleq\left\{\mathbf{v}: \mathbf{G V}_{1} \mathbf{u} \in \Lambda_{\mathbb{C}}\right.$ is the $k^{\text {th }}$ closest lattice point to $\left.\mathbf{y}\right\}$.

As shown in Fig. 1, the value of $D$ is determined by

$$
D=\left|S_{R_{\max }} \cap \Lambda_{\mathbb{C}}\right|
$$

where $S_{R_{\max }}$ is a sphere centered at $\mathbf{y}$ with radius

$$
R_{\max }\left(P_{\mathrm{v}}\right) \triangleq \max _{\|\mathbf{v}\|^{2} \leq P_{\mathbf{v}}}\|\mathbf{G Z} \mathbf{V}\|=\sqrt{\lambda_{\max } P_{\mathbf{v}}}
$$

where $\lambda_{\max }$ is the largest eigenvalue of $(\mathbf{G Z})^{H}(\mathbf{G Z})$.

Assuming $\mathbf{v} \in S_{k}, 1 \leq k \leq D$, the signal model (24) can be further viewed as an encryption algorithm that encrypts $\mathbf{u}$ to $\mathbf{y}$ using a one time pad secret key $\mathbf{v}$, such that $\mathbf{G V}_{1} \mathbf{u}$ is the $k^{\text {th }}$ closest lattice point to $\mathbf{y}$.

The security problem lies in how much Eve knows about $k$. The value of $k$ is uniquely determined by the vector $\tilde{\mathbf{n}}_{\mathrm{v}}$. Since we assume that the realizations of $\mathbf{G}$ and $\mathbf{Z}$ are known to Eve, $k$ is a function of $\mathbf{v}$. Since $\mathbf{v}$ is randomly and independently selected by Alice and is never disclosed to anyone, Eve can neither know its realization nor its distribution. Thus, given $\mathbf{y}$, Eve is not able to estimate the distribution of the index $k$.

Remark 2: The index $k$ can be interpreted as the effective one-time pad secret key, whose randomness comes from the artificial noise. The effective key space size is $D$.

From Eve's perspective, we assume that she knows $P_{\mathrm{v}}$, $R_{\max }\left(P_{\mathrm{v}}\right), D$ and the encryption process (24). Based on the above analysis, given $\mathbf{y}$, Eve only knows that $\mathbf{G V}_{1} \mathbf{u} \in$ $S_{R_{\max }} \cap \Lambda_{\mathbb{C}}$. Therefore, the posterior probability that Eve obtains $\mathbf{u}$, or equivalently, finds $k$, from the cryptogram $\mathbf{y}$, is equal to

$$
\operatorname{Pr}\{\mathbf{u} \mid \mathbf{y}\}=\operatorname{Pr}\{k \mid \mathbf{y}\}=\operatorname{Pr}\{\mathbf{u} \mid \mathbf{u} \in \mathcal{U}\}
$$

where

$$
\mathcal{U} \triangleq\left\{\mathbf{u}^{\prime}: \mathbf{G V}_{1} \mathbf{u}^{\prime} \in S_{R_{\max }} \cap \Lambda_{\mathbb{C}}\right\},
$$

and $|\mathcal{U}|=D$.
For any $\mathbf{u}^{\prime} \in \mathcal{U}$, using Bayes' theorem, we have

$$
\begin{aligned}
\operatorname{Pr}\left\{\mathbf{u}=\mathbf{u}^{\prime} \mid \mathbf{u} \in \mathcal{U}\right\} & =\frac{\operatorname{Pr}\left\{\mathbf{u}=\mathbf{u}^{\prime}\right\} \operatorname{Pr}\left\{\mathbf{u} \in \mathcal{U} \mid \mathbf{u}=\mathbf{u}^{\prime}\right\}}{\operatorname{Pr}\{\mathbf{u} \in \mathcal{U}\}} \\
& =\frac{\operatorname{Pr}\left\{\mathbf{u}=\mathbf{u}^{\prime}\right\}}{\operatorname{Pr}\{\mathbf{u} \in \mathcal{U}\}} .
\end{aligned}
$$

From (30) and (32), Eve's equivocation is given by

$$
H(\mathbf{u} \mid \mathbf{y})=H(k \mid \mathbf{y})=\sum_{\mathbf{u}^{\prime} \in \mathcal{U}} \frac{\operatorname{Pr}\left\{\mathbf{u}=\mathbf{u}^{\prime}\right\}}{\operatorname{Pr}\{\mathbf{u} \in \mathcal{U}\}} \log \frac{\operatorname{Pr}\{\mathbf{u} \in \mathcal{U}\}}{\operatorname{Pr}\left\{\mathbf{u}=\mathbf{u}^{\prime}\right\}} .
$$

Since

$$
\operatorname{Pr}\{\mathbf{u} \in \mathcal{U}\}=\sum_{\mathbf{u}^{\prime} \in \mathcal{U}} \operatorname{Pr}\left\{\mathbf{u}=\mathbf{u}^{\prime}\right\}
$$

the security level is determined by the cardinality of the set $\mathcal{U}$, or more specifically, by the value of $D$ :

1) if $D=1$, then $\operatorname{Pr}\{\mathbf{u} \in \mathcal{U}\}=\operatorname{Pr}\left\{\mathbf{u}=\mathbf{u}^{\prime}\right\}$, so that

$$
H(k \mid \mathbf{y})=H(\mathbf{u} \mid \mathbf{y})=0 . \quad \text { (no security) }
$$

2) if $D \geq 2$, then $\operatorname{Pr}\{\mathbf{u} \in \mathcal{U}\}>\operatorname{Pr}\left\{\mathbf{u}=\mathbf{u}^{\prime}\right\}$, so that

$$
H(k \mid \mathbf{y})=H(\mathbf{u} \mid \mathbf{y})>0 . \quad \text { (ideal secrecy) }
$$

3) as $D \rightarrow \infty$, then $\operatorname{Pr}\{\mathbf{u} \in \mathcal{U}\} \rightarrow 1$, so that

$$
H(k \mid \mathbf{y})=H(\mathbf{u} \mid \mathbf{y})=H(\mathbf{u}) . \quad \quad \text { (perfect secrecy) }
$$

Remark 3: Different from Shannon's one-time pad cryptosystem, the effective one-time pad secret key $k$ is not shared between Alice and Bob. In particular, it is independently generated by Alice, but not needed by Bob to decipher, while it is fully affecting Eve's ability to decipher the original message. This motivates the name of this cryptosystem as Unshared Secret Key (USK) cryptosystem.

\section{B. Analyzing Eve's Equivocation}

As shown in (33), Eve's equivocation lies in the value of $D$, which is known to Eve but not to Alice. We then estimate the value of $D$ from Alice's perspective. According to (26) and (27), $D$ is a function of $P_{\mathrm{v}}, \mathbf{H}$, and $\mathbf{G}$. Alice knows only $P_{\mathrm{v}}$ and $\mathbf{H}$, while regarding $\mathbf{G}$, she knows the statistics, but doesn't know the realization. Although Alice cannot know the exact value of $D$, she is able to estimate its cumulative distribution function (cdf), denoted by

$$
F_{D}\left(d, P_{\mathrm{v}}\right) \triangleq \operatorname{Pr}\{D<d\}
$$

where $d$ is a positive integer.

In the next section, we will show that Alice can ensure $F_{D}\left(d, P_{\mathrm{v}}\right) \rightarrow 0$ by increasing $P_{\mathrm{v}}$, i.e., she can guarantee that $D \geq d$, for any given $d$. 


\section{The SECURITY OF USK With INFINITE CONSTELLATIONS}

In this section, we show that the USK with infinite constellations provides Shannon's ideal secrecy and perfect secrecy. To prove the main theorems, we first introduce some lemmas.

We first define

$$
\begin{gathered}
\kappa(d) \triangleq d^{1 /\left(2 N_{\mathrm{E}}\right)} / \sqrt{\pi}, \\
\Delta(d) \triangleq \frac{\kappa(d)^{2 N_{\mathrm{E}}} \operatorname{vol}\left(\Lambda_{\mathbb{C}}\right)}{P_{\mathrm{V}}^{N_{\mathrm{E}}}},
\end{gathered}
$$

where $d$ is an integer and

$$
\operatorname{vol}\left(\Lambda_{\mathbb{C}}\right)=\left|\operatorname{det}\left(\left(\mathbf{G V}_{1}\right)^{H}\left(\mathbf{G V}_{1}\right)\right)\right| .
$$

Here, $\mathbf{G}$ is a complex Gaussian random matrix, while $\mathbf{V}_{1}$ is deterministic. Thus, $\Delta(d)$ is a random variable from Alice perspective. The following two lemmas are used to evaluate $F_{D}\left(d, P_{\mathrm{v}}\right)$ in $(35)$.

Lemma 1: If $P_{\mathrm{v}} \geq \rho^{2} / \Phi^{2 N_{\mathrm{B}} / N_{\mathrm{E}}}$ and $\rho>\kappa(d)$, then $\Delta(d) \stackrel{\text { a.s. }}{\rightarrow} 0$ as $N_{\mathrm{B}} \rightarrow \infty$, or equivalently,

$$
\operatorname{Pr}\left\{\Delta(d)>\left(\frac{\rho}{\kappa(d)}\right)^{-N_{\mathrm{B}}}\right\}<O\left(\left(\frac{\rho}{\kappa(d)}\right)^{-N_{\mathrm{B}}}\right)
$$

where

$$
\Phi=\left[\frac{\left(N_{\mathrm{E}}-N_{\mathrm{B}}\right) !}{N_{\mathrm{E}} !}\right]^{\frac{1}{2 N_{\mathrm{B}}}} .
$$

Proof: See Appendix A.

We next provide a more accurate expression of the tail distribution of $\Delta(d)$ for finite $N_{\mathrm{B}}$.

Lemma 2: If $P_{\mathrm{v}} \geq \rho^{2} / \Phi^{2 N_{\mathrm{B}} / N_{\mathrm{E}}}$ and $\rho>\kappa(d)$, then

$$
\operatorname{Pr}\left\{\Delta(d)>\left(\frac{\rho}{\kappa(d)}\right)^{-N_{\mathrm{B}}}\right\}<\Upsilon\left(\frac{\rho}{\kappa(d)}\right),
$$

where $\kappa(d)$ is given in (36), $\Phi$ is given (40), and

$$
\Upsilon(x)=\sum_{i=1}^{N_{\mathrm{B}}}\left(x e^{1-x}\right)^{N_{\mathrm{E}}-i+1} .
$$

Proof: See Appendix B.

Remark 4: From (42), it is easy to see that $\Upsilon(x)$ is monotonically decreasing function. Let

$$
N \triangleq N_{\mathrm{E}}-N_{\mathrm{B}}+1,
$$

then, as $x \rightarrow \infty$, we have

$$
\Upsilon(x)=O\left(\left(x^{-1} e^{x}\right)^{-N}\right)=O\left(e^{-x N}\right) .
$$

Lemmas 1 and 2 enable us to prove the following lemma.

Lemma 3: If $P_{\mathrm{v}} \geq \rho^{2} / \Phi^{2 N_{\mathrm{B}} / N_{\mathrm{E}}}$ and $\rho>\kappa(d), F_{D}\left(d, P_{\mathrm{v}}\right) \rightarrow 0$ as $N_{\mathrm{B}} \rightarrow \infty$, or equivalently,

$$
F_{D}\left(d, P_{\mathrm{v}}\right)<O\left(\left(\frac{\rho}{\kappa(d)}\right)^{-N_{\mathrm{B}}}\right),
$$

and for finite $N_{\mathrm{B}}$, we have

$$
F_{D}\left(d, P_{\mathrm{v}}\right)<\left(\frac{\rho}{\kappa(d)}\right)^{-N_{\mathrm{B}}}+\Upsilon\left(\frac{\rho}{\kappa(d)}\right),
$$

where $\kappa(d)$ is given in (36), $\Phi$ is given in (40), and $\Upsilon(x)$ is given in (42).

Proof: See Appendix C.

\section{A. Achieving Ideal Secrecy}

From (19) and the discussion following (33), ideal secrecy is achieved when $D \geq 2$. Lemma 3 enable us to prove the following equivalent theorem about achieving ideal secrecy.

Theorem 1: If $P_{\mathrm{v}}>\kappa(d)^{2} / \Phi^{2 N_{\mathrm{B}} / N_{\mathrm{E}}}$ and $d \geq 2$, as $N_{\mathrm{B}} \rightarrow \infty$,

$$
D \stackrel{a . s .}{\geq} d
$$

where $\kappa(d)$ is given in (36) and $\Phi$ is given in (40).

Proof: From (35) and (45), it is straightforward to see that $\operatorname{Pr}(D<d) \rightarrow 0$ as $N_{\mathrm{B}} \rightarrow \infty$.

Theorem 1 shows that for the USK, Eve cannot find a unique solution $\mathbf{u}$, since $D$ is almost surely greater than 2 .

We next estimate the secrecy outage probability when $N_{\mathrm{B}}$ is finite, defined by

$$
P_{\text {out }}(d) \triangleq \operatorname{Pr}\{D<d\},
$$

for any $d \geq 2$.

Theorem 2: Let $N_{\min }=\min \left\{N, N_{\mathrm{B}}\right\}$, where $N$ is given in (43). If

$$
P_{\mathrm{v}} \geq \varepsilon^{-2 / N_{\min }} \kappa(d)^{2} / \Phi^{2 N_{\mathrm{B}} / N_{\mathrm{E}}}
$$

and $d \geq 2$, then

$$
P_{\text {out }}(d)<O(\varepsilon),
$$

for any arbitrarily small $\varepsilon>0$, i.e., ideal secrecy is achieved with probability $1-O(\varepsilon)$, where $\kappa(d)$ is given in (36) and $\Phi$ is given in (40).

Proof: See Appendix D.

Theorem 2 shows that for finite $N_{\mathrm{B}}$, the outage of ideal secrecy can be made arbitrarily small by increasing $P_{\mathrm{v}}$.

Example 1: Let us apply Theorem 2 to the analysis of a USK scheme with $N_{\mathrm{A}}=9, N_{\mathrm{B}}=4, N_{\mathrm{E}}=8, \sigma_{\mathrm{E}}^{2}=0$, and

$$
P_{\mathrm{V}}=\varepsilon^{-2 / N_{\min }} \kappa(d)^{2} / \Phi^{2 N_{\mathrm{B}} / N_{\mathrm{E}}} .
$$

We evaluate the secrecy outage probability in (48) for the $i^{\text {th }}$ channel use. We generate 50000 pairs of mutually independent complex Gaussian random matrices $\{\mathbf{G}, \mathbf{H}\}$. For each pair of $\{\mathbf{G}, \mathbf{H}\}$, we evaluate the corresponding realization $\tilde{D}$ of the random variable $D$ by

$$
\tilde{D} \approx \frac{\operatorname{vol}\left(S_{R_{\max }}\right)}{\operatorname{vol}\left(\Lambda_{\mathbb{C}}\right)}=\left(\frac{R_{\max }\left(P_{\mathrm{v}}\right)}{r_{\mathrm{eff}}\left(\Lambda_{\mathbb{C}}\right)}\right)^{2 N_{\mathrm{B}}},
$$

where $r_{\text {eff }}\left(\Lambda_{\mathbb{C}}\right)$ is given in (21), $R_{\max }\left(P_{\mathrm{v}}\right)$ is given in (29). Based on the corresponding 50000 realizations of $D$, we compute the probability of $D<d$, i.e., $P_{\text {out }}(d)$. Fig. 2 shows the 


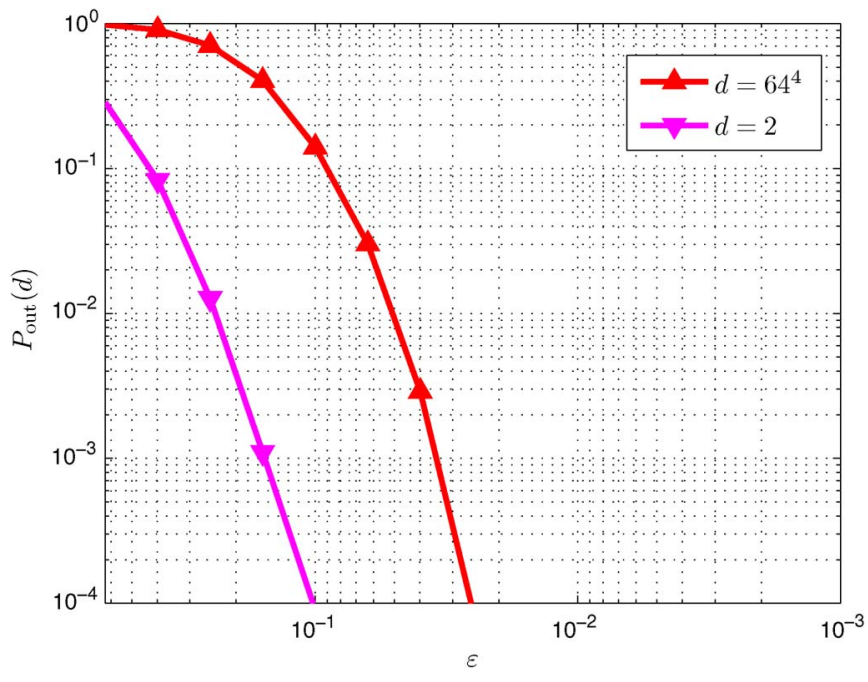

Fig. 2. $\quad P_{\text {out }}(d)$ vs. $\varepsilon$ with $N_{\mathrm{A}}=9, N_{\mathrm{B}}=4$, and $N_{\mathrm{E}}=8$.

value of $P_{\text {out }}(d)$ as a function of $\varepsilon$, with $d=2$ and $d=64^{4}$ (large number), respectively. As expected, the value of $P_{\text {out }}(d)$ decreases with decreasing $\varepsilon$, or equivalently, increasing $P_{\mathrm{v}}$.

\section{B. Achieving Perfect Secrecy}

From (20), perfect secrecy requires

$$
H(\mathbf{u} \mid \mathbf{y})=H(\mathbf{u}) .
$$

According to (33), the problem then reduces to ensuring $D \rightarrow \infty$. From Theorems 1 and 2, achieving perfect secrecy requires infinite AN peak power $P_{\mathrm{v}}$, which is of theoretical interest only.

\section{Unshared SeCRet Key CRyptosystem With Finite CONSTELlations}

In this section, we show that the idea of USK can be applied to practical systems using finite constellations. In this case, we define the concept of secrecy outage and define a secrecy outage probability. We will later show how such probability can be made arbitrarily small by considering either longer blocks of messages or larger constellation size.

\section{A. Encryption}

We consider a sequence of $K$ mutually independent messages $\left\{\mathbf{m}_{l}\right\}_{1}^{K}$, where each one contains $n$ mutually independent information bits. For each $\mathbf{m}$, Alice maps the corresponding $n$ bits to $N_{\mathrm{B}}$ elements of $\mathbf{u}$ for $B$ channel uses. Each elements of $\mathbf{u}$ is uniformly selected from a $M$-QAM constellation $\tilde{\mathcal{Q}}$, where $\Re(\tilde{\mathcal{Q}})=\Im(\tilde{\mathcal{Q}})=\{0,1, \ldots, \sqrt{M}-1\}$. We ignore the shifting and scaling operations at Alice to minimize the transmit power. Consequently, we have

$$
n=B N_{\mathrm{B}} \log _{2} M .
$$

Let $\left\{\mathbf{u}_{i}\right\}_{1}^{B}$ be the block of transmitted vectors corresponding to one message $\mathbf{m}$.

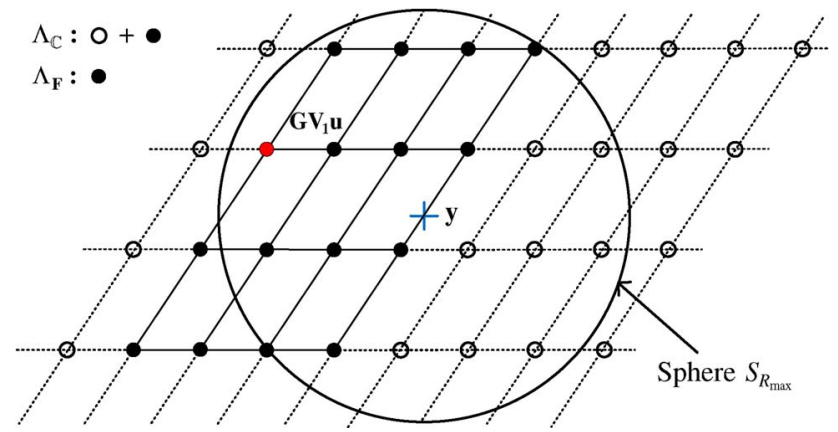

Fig. 3. The USK cryptosystem with finite constellations.

To secure the total $C=K B$ transmitted vectors $\left\{\mathbf{u}_{j}\right\}_{1}^{C}$, Alice enciphers $\left\{\mathbf{u}_{j}\right\}_{1}^{C}$ into the cryptograms $\left\{\mathbf{y}_{j}\right\}_{1}^{C}$ using a sequence of mutually independent keys $\left\{\mathbf{v}_{j}\right\}_{1}^{C}$. Across the $C$ channel uses, we assume that $\left\{\mathbf{v}_{j}\right\}_{1}^{C}$ and $\left\{\mathbf{u}_{j}\right\}_{1}^{C}$ are mutually independent, and $\left\{\mathbf{G}_{j}\right\}_{1}^{C}$ are mutually independent Gaussian random matrices. No assumption is needed about the statistics of $\left\{\mathbf{H}_{j}\right\}_{1}^{C}$, since its realization is known to Alice and Eve.

Since $\left\{\mathbf{v}_{j}\right\}_{1}^{C}$ and $\left\{\mathbf{u}_{j}\right\}_{1}^{C}$ are mutually independent, using (19), we only need to demonstrate the encryption process for one block of transmitted vectors $\left\{\mathbf{u}_{i}\right\}_{1}^{B}$ corresponding to a message $\mathbf{m}$.

The encryption process is the same as that of the infinite constellation case: for the $i^{\text {th }}$ channel use, Alice independently chooses a one time pad key $\mathbf{v}_{i}$ from the set $S$ in (22), and encrypts $\mathbf{u}_{i}$ to $\mathbf{y}_{i}$ in (24) using $\mathbf{v}_{i}$, such that $\mathbf{G}_{i} \mathbf{V}_{1, i} \mathbf{u}_{i}$ is the $k_{i}^{\text {th }}$ closest lattice point to $\mathbf{y}_{i}$, within the infinite lattice

$$
\Lambda_{\mathbb{C}, i}=\left\{\mathbf{G}_{i} \mathbf{V}_{1, i} \mathbf{u}, \mathbf{u} \in \mathbb{Z}[i]^{N_{\mathrm{B}}}\right\} .
$$

The value of $k_{i}$ ranges from 1 to $D_{i}$, where

$$
D_{i}=\left|S_{R_{\max , i}} \cap \Lambda_{\mathbb{C}, i}\right|,
$$

and $S_{R_{\max , i}}$ is a sphere centered at $\mathbf{y}_{i}$ with radius:

$$
R_{\max , i}\left(P_{\mathrm{v}}\right) \triangleq \max _{\left\|\mathbf{v}_{i}\right\|^{2} \leq P_{\mathbf{v}}}\left\|\mathbf{G}_{i} \mathbf{Z}_{i} \mathbf{v}_{i}\right\|=\sqrt{\lambda_{\max , i} P_{\mathbf{v}}}
$$

where $\lambda_{\max , i}$ is the largest eigenvalue of $\left(\mathbf{G}_{i} \mathbf{Z}_{i}\right)^{H}\left(\mathbf{G}_{i} \mathbf{Z}_{i}\right)$. As shown in Fig. 3, $D_{i}$ represents the total number of points within the sphere $S_{R_{\max , i}}$.

Different from the infinite constellation case, the condition $D_{i} \geq 2$ in (33) cannot ensure $H\left(\mathbf{u}_{i} \mid \mathbf{y}_{i}\right)>0$. The reason is that Eve knows that $\mathbf{G}_{i} \mathbf{V}_{1, i} \mathbf{u}_{i}$ is a finite lattice constellation, i.e., a finite subset of $\Lambda_{\mathbb{C}, i}$ :

$$
\Lambda_{\mathrm{F}, i} \triangleq\left\{\mathbf{G}_{i} \mathbf{V}_{1, i} \mathbf{u}, \mathbf{u} \in \tilde{\mathcal{Q}}^{N_{\mathrm{B}}}\right\} .
$$

Since $k_{i}$ is a function of $\mathbf{v}_{i}$, which is randomly and independently selected by Alice and is never disclosed to anyone, Eve can neither know the distribution of $k_{i}$. Given $\mathbf{y}_{i}$, Eve only knows that $\mathbf{G}_{i} \mathbf{V}_{1, i} \mathbf{u}_{i} \in S_{R_{\max , i}} \cap \Lambda_{\mathrm{F}, i}$. Let $L_{i}$ be the cardinality of such choices, i.e.,

$$
L_{i}=\left|S_{R_{\max , i}} \cap \Lambda_{\mathrm{F}, i}\right| .
$$


Since $\Lambda_{\mathrm{F}, i} \subset \Lambda_{\mathbb{C}, i}$, we have

$$
1 \leq L_{i} \leq D_{i} .
$$

As shown in Fig. 3, $L_{i}$ represents the number of solid points within the sphere $S_{R_{\max , i}}$.

Remark 5: Due to the use of finite constellation $\tilde{\mathcal{Q}}^{N_{\mathrm{B}}}$, we redefine the effective secrecy key $k_{i}$ as $k_{\mathrm{F}, i}$, that is, $\mathbf{G}_{i} \mathbf{V}_{1, i} \mathbf{u}_{i}$ is the $k_{\mathrm{F}, i}^{\mathrm{th}}$ closest lattice point to $\mathbf{y}_{i}$, within the finite lattice constellation $\Lambda_{\mathrm{F}, i}$. The corresponding key space size is $L_{i}$ per channel use.

Remark 6: The practical secrecy scheme [21] is a special case of USK cryptosystem with $k_{\mathrm{F}, i} \geq 2$.

\section{B. Analyzing Eve's Equivocation}

We then show that Eve's equivocation $H\left(\left\{\mathbf{u}_{i}\right\}_{1}^{B} \mid\left\{\mathbf{y}_{i}\right\}_{1}^{B}\right)$ is determined by $\left\{L_{i}\right\}_{1}^{B}$. The posterior probability that Eve obtains $\mathbf{u}_{i}$, or equivalently, finds $k_{\mathrm{F}, i}$, is equal to

$$
\operatorname{Pr}\left\{\mathbf{u}_{i} \mid \mathbf{y}_{i}\right\}=\operatorname{Pr}\left\{k_{\mathrm{F}, i} \mid \mathbf{y}_{i}\right\}=\operatorname{Pr}\left\{\mathbf{u}_{i} \mid \mathbf{u}_{i} \in \mathcal{U}_{\mathrm{F}, i}\right\},
$$

where

$$
\mathcal{U}_{\mathrm{F}, i} \triangleq\left\{\mathbf{u}^{\prime}: \mathbf{G}_{i} \mathbf{V}_{1, i} \mathbf{u}^{\prime} \in S_{R_{\max , i}} \cap \Lambda_{\mathrm{F}, i}\right\} .
$$

Due to the use of uniform constellation $\tilde{\mathcal{Q}}^{N_{\mathrm{B}}}$, according to Bayes' theorem, we have

$$
\operatorname{Pr}\left\{\mathbf{u}_{i} \mid \mathbf{u}_{i} \in \mathcal{U}_{\mathrm{F}, i}\right\}=\frac{1}{L_{i}} .
$$

To recover one message $\mathbf{m}$, Eve has to recover all vectors in $\left\{\mathbf{u}_{i}\right\}_{1}^{B}$, or equivalently, find $\left\{k_{\mathrm{F}, i}\right\}_{1}^{B}$. Therefore, Eve's equivocation is given by

$H\left(\mathbf{m} \mid\left\{\mathbf{y}_{i}\right\}_{1}^{B}\right)=H\left(\left\{k_{\mathrm{F}, i}\right\}_{1}^{B} \mid\left\{\mathbf{y}_{i}\right\}_{1}^{B}\right)=H\left(\left\{\mathbf{u}_{i}\right\}_{1}^{B} \mid\left\{\mathbf{y}_{i}\right\}_{1}^{B}\right)$.

Moreover, since $\mathbf{u}_{i}$ is independent of $\mathbf{u}_{j}$ and $\mathbf{y}_{j}$, we have

$$
H\left(\left\{\mathbf{u}_{i}\right\}_{1}^{B} \mid\left\{\mathbf{y}_{i}\right\}_{1}^{B}\right)=\sum_{i=1}^{B} H\left(\mathbf{u}_{i} \mid \mathbf{y}_{i}\right)=\sum_{i=1}^{B} \log L_{i} .
$$

\section{Ideal Secrecy Outage}

Based on (65), Eve's equivocation is dominated by the values in $\left\{L_{i}\right\}_{1}^{B}$, which are known to Eve. From Alice's perspective, according to (59) and (62), $L_{i}$ is a function of $\mathbf{G}_{i}$, thus a random variable. Although Alice cannot know the exact values in $\left\{L_{i}\right\}_{1}^{B}$, she may be able to evaluate the cdf of Eve's equivocation, given by

$$
\begin{aligned}
\operatorname{Pr}\left\{\sum_{i=1}^{B} \log L_{i}<\log d\right\} & \leq \operatorname{Pr}\left\{\log L_{i}<\log d, 1 \leq i \leq B\right\} \\
& =\operatorname{Pr}\left\{L_{1}<d, \ldots, L_{B}<d\right\} \\
& \triangleq P_{\mathrm{F}, \text { out }}(d, B)
\end{aligned}
$$

where $2 \leq d \leq M^{N_{\mathrm{B}}}$.
We refer to the event

$$
\sum_{i=1}^{B} \log L_{i}<\log d
$$

as the secrecy outage due to the use of the finite constellation $\tilde{\mathcal{Q}}^{N_{\mathrm{B}}}$. We refer to $P_{\mathrm{F}, \text { out }}(d, B)$ as the secrecy outage probability. From (65) and (66), if $P_{\mathrm{F} \text {,out }}(d, B) \rightarrow 0$,

$$
H\left(\left\{\mathbf{u}_{i}\right\}_{1}^{B} \mid\left\{\mathbf{y}_{i}\right\}_{1}^{B}\right)=H\left(\left\{k_{\mathrm{F}, i}\right\}_{1}^{B} \mid\left\{\mathbf{y}_{i}\right\}_{1}^{B}\right) \geq \log d .
$$

In the next section, we will show that Alice can ensure $P_{\mathrm{F}, \text { out }}(d, B) \rightarrow 0$ by increasing the message block size $B$ with certain $M$ and $P_{\mathrm{v}}$.

\section{ThE SECURITY OF USK With Finite CONSTELlations}

In this section, we show that the USK with the finite constellation $\tilde{\mathcal{Q}}^{N_{\mathrm{B}}}$ provides Shannon's ideal secrecy with an arbitrarily small outage. To prove the main theorems, we first introduce the following lemma.

We define

$$
\Theta\left(P_{\mathrm{v}}\right) \triangleq \frac{2 R_{\max }\left(P_{\mathrm{v}}\right)}{\sqrt{\bar{M}} r_{\mathrm{eff}}\left(\Lambda_{\mathbb{C}}\right)}
$$

where $r_{\text {eff }}\left(\Lambda_{\mathbb{C}}\right)$ is given in (21) and $R_{\max }\left(P_{\mathrm{v}}\right)$ is given in (57). From Alice perspective, $\Theta\left(P_{\mathrm{v}}\right)$ is a function of $\mathbf{G}$, thus is a random variable. Its cdf is bounded by the following lemma.

Lemma 4:

$\operatorname{Pr}\left\{\Theta\left(P_{\mathrm{v}}\right)<x\right\}$

$$
\left.>\prod_{j=1}^{N_{\mathrm{B}}} B \frac{N_{\mathrm{E}}\left(N_{\mathrm{A}}-N_{\mathrm{B}}\right) g(x, j)}{B_{N_{\mathrm{E}}\left(N_{\mathrm{A}}-N_{\mathrm{B}}\right)}, N_{\mathrm{E}}-j+1}\right),\left(\frac{N_{\mathrm{E}}\left(N_{\mathrm{A}}-N_{\mathrm{B}}\right) g(x, j)+N_{\mathrm{E}}-j+1}{N^{\prime}}\right),
$$

where

$$
g(x, j)=\frac{x^{2} M N_{\mathrm{B}}\left(N_{\mathrm{E}}-j+1\right)}{4 \pi e P_{\mathrm{v}} N_{\mathrm{E}}\left(N_{\mathrm{A}}-N_{\mathrm{B}}\right)},
$$

and $B_{a, b}(x)$ is the regularized incomplete beta function [27]:

$$
B_{a, b}(x) \triangleq \sum_{j=a}^{a+b-1}\left(\begin{array}{c}
a+b-1 \\
j
\end{array}\right) x^{j}(1-x)^{a+b-1-j} .
$$

Proof: See Appendix E.

\section{A. Achieving Ideal Secrecy}

As shown in (19) and (65), ideal secrecy is achieved when $\sum_{i=1}^{B} \log L_{i}>0$. From (66), the problem then reduces to ensuring

$$
P_{\mathrm{F}, \text { out }}(d, B) \rightarrow 0,
$$

for any $d \geq 2$. Lemma 4 enables us to prove the following theorem. 


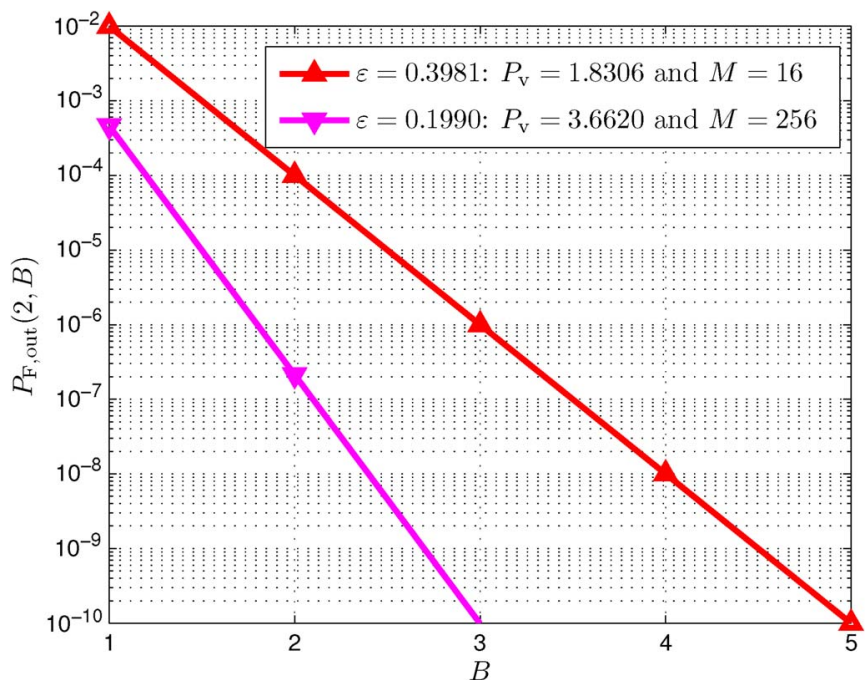

Fig. 4. $\quad P_{\mathrm{F} \text {,out }}(2, B)$ vs. $M$ and $B$ with $N_{\mathrm{A}}=4, N_{\mathrm{B}}=2$, and $N_{\mathrm{E}}=3$.

Theorem 3: If $\varepsilon<1, d \geq 2, P_{\mathrm{v}}=\varepsilon^{-2 / N_{\min }} \kappa(d)^{2} / \Phi^{2 N_{\mathrm{B}} / N_{\mathrm{E}}}$, and $M \geq \varepsilon^{-3-2 / N_{\min }} \kappa(d)^{2}$, then

$$
P_{\mathrm{F}, \text { out }}(d, B)<O\left(\varepsilon^{B}\right),
$$

where $\kappa(d)$ is given in (36) and $\Phi$ is given in (40), i.e., ideal secrecy is achieved with probability $1-O\left(\varepsilon^{B}\right)$.

Proof: See Appendix F.

Theorem 3 shows that for finite $N_{\mathrm{B}}$ and finite constellation $\tilde{\mathcal{Q}}^{N_{\mathrm{B}}}$, the ideal secrecy outage can be made arbitrarily small. Given a desired pair $\{\varepsilon, d\}$, Alice can easily compute the required values of $P_{\mathrm{v}}$ and $M$ to realize the USK cryptosystem.

Example 2: We consider a USK scheme with $N_{\mathrm{A}}=4$, $N_{\mathrm{B}}=2, N_{\mathrm{E}}=3$, and $\sigma_{\mathrm{E}}^{2}=0$. To apply Theorem 3 , we fix $d=2$ and consider two cases where $\varepsilon=0.3981$ and 0.1990 . The conditions in Theorem 3 then reduce to

$$
\begin{aligned}
& P_{\mathrm{v}}=1.8306 \text { and } M \geq 15.9659, \text { for } \varepsilon=0.3981, \\
& P_{\mathrm{v}}=3.6620 \text { and } M \geq 255.7297, \text { for } \varepsilon=0.1990 .
\end{aligned}
$$

Fig. 4 compares the value of $P_{\mathrm{F} \text {,out }}(2, B)$ as a function of $B$. Note that $P_{\mathrm{F} \text {,out }}(2, B)$ can be written as

$$
\operatorname{Pr}\left\{L_{1}=1, \ldots, L_{B}=1\right\}=\operatorname{Pr}\left\{\sum_{i=1}^{B} \log L_{i}=0\right\} .
$$

We observe that $P_{\mathrm{F} \text {, out }}(2, B)=4.6250 \times 10^{-4}$ when $P_{\mathrm{v}}=$ $3.6620, M=256$, and $B=1$. It confirms that the secrecy outage probability can be made arbitrarily small by increasing $P_{\mathrm{v}}$ and $M$. Meanwhile, we observe that the secrecy outage probability decreases exponentially with $B$.

Remark 7: For the finite constellation case, the value of target equivocation at Eve is given by $\log d$ in (68). Note that this is not easily computable for the infinite constellation case according to (33).

\section{B. Peak AN-to-Signal Power Ratio}

By shifting and scaling, $\mathbf{u} \in \tilde{\mathcal{Q}}^{N_{\mathrm{B}}}$ can be converted into a regular $M$-QAM symbol $\overline{\mathbf{u}} \in \mathcal{Q}^{N_{\mathrm{B}}}$. To measure the power efficiency of the proposed USK cryptosystem, we define

$$
r \triangleq \frac{P_{\mathrm{v}}}{\mathrm{E}\left(\left\|\mathbf{V}_{1} \overline{\mathbf{u}}\right\|^{2}\right)}
$$

as the ratio of the peak $\mathrm{AN}$ power $P_{\mathrm{v}}$ and the average transmitted signal power.

Since

$$
\mathrm{E}\left(\left\|\mathbf{V}_{1} \overline{\mathbf{u}}\right\|^{2}\right)=\mathrm{E}\left(\|\overline{\mathbf{u}}\|^{2}\right)=\frac{2(M-1) N_{\mathrm{B}}}{3},
$$

the corresponding ratio as a function of $P_{\mathrm{v}}$ is given by

$$
r=\frac{3 P_{\mathrm{v}}}{2(M-1) N_{\mathrm{B}}} .
$$

Example 3: Under the same setting of Example 2, if $M=256, r=1.08 \%$. We see that the proposed USK cryptosystem is very practical, since it requires a very small proportion of the total transmission power. Note that the value of $r$ can be further reduced by increasing the constellation size $M$.

\section{DISCUSSIONS}

\section{A. USK Cryptosystems vs. Previous AN Based Schemes}

The existing AN based security schemes [19], [28], [29] leverage infinite-length wiretap codes, where the aim is to achieve strong secrecy.

In contrast, the proposed USK cryptosystem is valid for any coded/uncoded MIMO with finite block length and QAM signaling. Our scheme achieves Shannon's ideal secrecy with an arbitrarily small outage probability.

\section{B. Extension to the Case of $N_{\mathrm{E}} \geq N_{\mathrm{A}}$}

The constraint $N_{\mathrm{E}}<N_{\mathrm{A}}$ is a common assumption that appears in the vast literature on AN based schemes [19], [28], [29]. Under this condition, we have shown the existence of an unshared secret key cryptosystem which provides Shannon's ideal secrecy.

If $N_{\mathrm{E}} \geq N_{\mathrm{A}}$, $\mathrm{G}$ has a left inverse, denoted by $\mathrm{G}^{\dagger}$, then Eve can remove the unshared secret key $\mathbf{v}$ by multiplying $\mathbf{y}$ by $\mathbf{W}=\mathbf{H G}^{\dagger}$, i.e.,

$$
\mathbf{W} \mathbf{y}=\mathbf{H V}_{1} \mathbf{u}+\mathbf{W} \mathbf{n}_{\mathrm{E}}
$$

We can show that this attack amplifies Eve's channel noise greatly. Consequently, $\mathbf{n}_{\mathrm{E}}$ takes the role of the unshared secret key. We can show that with certain amount of $\sigma_{\mathrm{E}}^{2}$, ideal secrecy is achievable. This result will be reported in our next paper.

\section{CONCLUSION}

We have exploited the role that artificial noise plays in physical layer security to show that it can be used as an unshared onetime pad secret key. The proposed unshared secret key (USK) cryptosystem with an infinite lattice input alphabet provides Shannon's ideal secrecy and perfect secrecy by tuning the power allocated to the artificial noise component. Moreover, 
unlike the traditional AN technique, this USK system can be applied to practical systems using finite lattice constellations. We have shown that ideal secrecy can be obtained with an arbitrarily small outage probability. Our results provide analytical insights relating cryptography and physical layer security on a fundamental level. Future work will generalize USK to relaying networks.

\section{APPENDIX}

\section{A. Proof of Lemma 1}

Recalling that

$$
\Delta(d)=\frac{\kappa(d)^{2 N_{\mathrm{E}}}\left|\operatorname{det}\left(\left(\mathbf{G} \mathbf{V}_{1}\right)^{H}\left(\mathbf{G} \mathbf{V}_{1}\right)\right)\right|}{P_{\mathrm{V}}^{N_{\mathrm{E}}}} .
$$

From Alice's perspective, G is a complex Gaussian random matrix. The matrix $\mathbf{V}_{1}$ with orthonormal columns is known. According to [30], $\mathbf{G V}_{1}$ a Gaussian random matrix with i.i.d. elements. Moreover, $\left|\operatorname{det}\left(\left(\mathbf{G V}_{1}\right)^{H}\left(\mathbf{G V}_{1}\right)\right)\right|$ can be expressed as the product of independent Chi-squared variables [31]:

$$
\left|\operatorname{det}\left(\left(\mathbf{G V}_{1}\right)^{H}\left(\mathbf{G} \mathbf{V}_{1}\right)\right)\right|=\prod_{i=1}^{N_{\mathrm{B}}} \frac{1}{2} \mathcal{X}^{2}\left(2\left(N_{\mathrm{E}}-i+1\right)\right) \text {. }
$$

Using the properties of the Chi-squared distribution and central limit theorem, as $N_{\mathrm{B}} \rightarrow \infty$, we have

$$
\frac{\sum_{i=1}^{N_{\mathrm{B}}} \log \mathcal{X}^{2}\left(2\left(N_{\mathrm{E}}-i+1\right)\right)-A}{\sqrt{V}} \stackrel{\text { a.s. }}{\rightarrow} \mathcal{N}(0,1),
$$

where

$$
\begin{aligned}
A & =\sum_{i=1}^{N_{\mathrm{B}}} \mathrm{E}\left(\log \mathcal{X}^{2}\left(2\left(N_{\mathrm{E}}-i+1\right)\right)\right), \\
V & =\sum_{i=1}^{N_{\mathrm{B}}} \operatorname{Var}\left(\log \mathcal{X}^{2}\left(2\left(N_{\mathrm{E}}-i+1\right)\right)\right) .
\end{aligned}
$$

Using the properties of Log Chi-squared distributions [32], we have

$$
\begin{aligned}
& A=\sum_{k=N_{\mathrm{E}}-N_{\mathrm{B}}+1}^{N_{\mathrm{E}}}(\log 2+\psi(k)), \\
& V=\sum_{k=N_{\mathrm{E}}-N_{\mathrm{B}}+1}^{N_{\mathrm{E}}} \psi_{1}(k),
\end{aligned}
$$

where $\psi(x)=\frac{d}{d x} \log \Gamma(x)$ is the digamma function, and $\psi_{1}(x)=\frac{d^{2}}{d x^{2}} \log \Gamma(x)$ is the trigamma function.

Informally, we may write (82) and (83) as

$$
\left|\operatorname{det}\left(\left(\mathbf{G V}_{1}\right)^{H}\left(\mathbf{G} \mathbf{V}_{1}\right)\right)\right| \approx 2^{-N_{\mathrm{B}}} e^{A+\mathcal{N}(0, V)}
$$

According to (84), as $N_{\mathrm{B}} \rightarrow \infty, \Delta(d)$ converges to the random variable $\Omega$ :

$$
\Omega \triangleq \frac{\kappa(d)^{2 N_{\mathrm{E}}} \exp (A+\mathcal{N}(0, V))}{2^{N_{\mathrm{B}}} P_{\mathrm{v}}^{N_{\mathrm{E}}}} .
$$

To simplify the expressions of $A$ and $V$, we use the following approximations [32]:

$$
\begin{aligned}
\psi(k) & \approx \log k-1 /(2 k), \\
\psi_{1}(k) & \approx 1 / k .
\end{aligned}
$$

Then, we have

$$
V \leq \sum_{i=1}^{N_{\mathrm{B}}} \frac{1}{k} \leq \log N_{\mathrm{B}}+\varsigma<\log 2 N_{\mathrm{B}}
$$

where $\varsigma$ is Euler-Mascheroni constant. Similarly, we have

$$
\begin{aligned}
& A=\sum_{k=N_{\mathrm{E}}-N_{\mathrm{B}}+1}^{N_{\mathrm{E}}}\left(\log 2+\log k-\frac{1}{2 k}\right) \\
& <N_{\mathrm{B}} \log 2+\log \Phi^{-2 N_{\mathrm{B}}} \text {, }
\end{aligned}
$$

where

$$
\Phi=\left[\frac{\left(N_{\mathrm{E}}-N_{\mathrm{B}}\right) !}{N_{\mathrm{E}} !}\right]^{\frac{1}{2 N_{\mathrm{B}}}} .
$$

From (88) and (85), $\Omega$ can be upper bounded by

$$
\Omega<\frac{\kappa(d)^{2 N_{\mathrm{E}}} \exp (\mathcal{N}(0, V))}{\Phi^{2 N_{\mathrm{B}}} P_{\mathrm{V}}^{N_{\mathrm{E}}}} .
$$

Recall that $N_{\mathrm{E}} \geq N_{\mathrm{B}}$. By substituting $P_{\mathrm{v}} \geq \rho^{2} / \Phi^{2 N_{\mathrm{B}} / N_{\mathrm{E}}}$ and $\rho>\kappa(d)$ to the right side of (90), we have

$$
\Omega<\frac{\exp (\mathcal{N}(0, V))}{(\rho / \kappa(d))^{2 N_{\mathrm{E}}}} \leq \frac{\exp (\mathcal{N}(0, V))}{(\rho / \kappa(d))^{2 N_{\mathrm{B}}}} \triangleq \Omega_{\mathrm{UB}},
$$

and

$$
\begin{aligned}
\operatorname{Pr} & \left\{\Delta(d)>(\rho / \kappa(d))^{-N_{\mathrm{B}}}\right\} \\
& <\operatorname{Pr}\left\{\Omega_{\mathrm{UB}}>(\rho / \kappa(d))^{-N_{\mathrm{B}}}\right\} \\
& =\operatorname{Pr}\left\{\mathcal{N}(0, V)>N_{\mathrm{B}} \log (\rho / \kappa(d))\right\} \\
& <1 / 2 \exp \left(-\frac{N_{\mathrm{B}}^{2} \log ^{2}(\rho / \kappa(d))}{2 V}\right) \\
& <1 / 2 \exp \left(-\frac{N_{\mathrm{B}}^{2} \log ^{2}(\rho / \kappa(d))}{2 \log ^{2} 2 N_{\mathrm{B}}}\right) \\
& =O\left((\rho / \kappa(d))^{-N_{\mathrm{B}}}\right),
\end{aligned}
$$

where $(a)$ holds because of (87).

From (92) and (81), if $\rho>\kappa(d)$, as $N_{\mathrm{B}} \rightarrow \infty$, we have $\Delta(d) \stackrel{\text { a.s. }}{\rightarrow} 0$.

\section{B. Proof of Lemma 2}

We recall (81) and (82) and consider the random variable

$$
\Psi \triangleq \prod_{i=1}^{N_{\mathrm{B}}} \frac{\mathcal{X}^{2}\left(2\left(N_{\mathrm{E}}-i+1\right)\right)}{2\left(N_{\mathrm{E}}-i+1\right)} .
$$

Recalling that $N_{\mathrm{E}} \geq N_{\mathrm{B}}$. By substituting $\Psi, P_{\mathrm{v}} \geq \rho^{2} / \Phi^{2 N_{\mathrm{B}}} / N_{\mathrm{E}}$, and $\rho>\kappa(d)$ to the right side of (81), we have

$$
\begin{aligned}
\Delta(d) & =(\rho / \kappa(d))^{-2 N_{\mathrm{E}}} \Psi \\
& \leq(\rho / \kappa(d))^{-2 N_{\mathrm{B}}} \Psi .
\end{aligned}
$$


Consequently, we obtain

$$
\begin{aligned}
\operatorname{Pr} & \left\{\Delta(d)>(\rho / \kappa(d))^{-N_{\mathrm{B}}}\right\} \\
& \leq \operatorname{Pr}\left\{\Psi(\rho / \kappa(d))^{-2 N_{\mathrm{B}}}>(\rho / \kappa(d))^{-N_{\mathrm{B}}}\right\} \\
& =\operatorname{Pr}\left\{\Psi>(\rho / \kappa(d))^{N_{\mathrm{B}}}\right\} \\
& \leq \operatorname{Pr}\left\{\sum_{i=1}^{a} \frac{\mathcal{X}^{2}\left(2\left(N_{\mathrm{E}}-i+1\right)\right)}{2\left(N_{\mathrm{E}}-i+1\right)}>N_{\mathrm{B}} \rho / \kappa(d)\right\} \\
& <\sum_{i=1}^{N_{\mathrm{B}}} \operatorname{Pr}\left\{\mathcal{X}^{2}\left(2\left(N_{\mathrm{E}}-i+1\right)\right) \geq 2\left(N_{\mathrm{E}}-i+1\right) \rho / \kappa(d)\right\} \\
& \leq \sum_{i=1}^{N_{\mathrm{B}}}\left(e^{1-\rho / \kappa(d)} \rho / \kappa(d)\right)^{N_{\mathrm{E}}-i+1} \\
& \triangleq \Upsilon(\rho / \kappa(d)),
\end{aligned}
$$

where $(a)$ holds due to the inequality of arithmetic and geometric means.

\section{Proof of Lemma 3}

We pick an element $\mathbf{v}_{0}$ from $\mathcal{S}$ with $\left\|\mathbf{v}_{0}\right\|^{2}=P_{\mathrm{v}}$. Suppose that $\mathbf{v}_{0} \in S_{k_{0}}$, where $k_{0}$ is the corresponding effective secret key. Since $D \geq k_{0}$, we have

$$
F_{D}\left(d, P_{\mathrm{v}}\right)=\operatorname{Pr}\{D<d\}<\operatorname{Pr}\left\{k_{0} \leq d\right\}
$$

The problem then reduces to evaluating $\operatorname{Pr}\left\{k_{0} \leq d\right\}$.

Let $\mathcal{S}_{R}$ be a sphere of radius $R \leq R_{\max }\left(P_{\mathrm{v}}\right)$ centered at $\mathbf{y}$, where $\operatorname{vol}\left(\mathcal{S}_{R}\right)=d \cdot \operatorname{vol}\left(\Lambda_{\mathbb{C}}\right)$ (see Fig. 1). Let $K$ be the number of the points in $\mathcal{S}_{R} \cap \Lambda_{\mathbb{C}}$. We have

$$
K \approx \frac{\operatorname{vol}\left(\mathcal{S}_{R}\right)}{\operatorname{vol}\left(\Lambda_{\mathbb{C}}\right)}=d
$$

If $\mathbf{G V}_{1} \mathbf{u} \in \mathcal{S}_{R}$, we have $k_{0} \leq d$, and vice versa. Thus, the two events are equivalent, i.e.,

$$
\operatorname{Pr}\left\{k_{0} \leq d\right\}=\operatorname{Pr}\left\{\mathbf{G V}_{1} \mathbf{u} \in \mathcal{S}_{R}\right\}
$$

Let $\mathcal{S}_{R}^{\prime}$ be a sphere with the same radius $R$ centered at $\mathbf{G V}_{1} \mathbf{u}$. If $\mathbf{G V}_{1} \mathbf{u} \in \mathcal{S}_{R}$, then $\mathbf{y} \in \mathcal{S}_{R}^{\prime}$, and vice versa. Thus, the two events are equivalent, i.e.,

$$
\operatorname{Pr}\left\{\mathbf{G V}_{1} \mathbf{u} \in \mathcal{S}_{R}\right\}=\operatorname{Pr}\left\{\mathbf{y} \in \mathcal{S}_{R}^{\prime}\right\} .
$$

From (96), (98), and (99), we have

$$
\begin{aligned}
F_{D}( & \left.d, P_{\mathrm{v}}\right) \\
< & \operatorname{Pr}\left\{\mathbf{y} \in \mathcal{S}_{R}^{\prime}\right\} \\
= & \operatorname{Pr}\left\{\mathbf{y} \in \mathcal{S}_{R}^{\prime} \mid \operatorname{vol}\left(\mathcal{S}_{R}^{\prime}\right) \leq C\right\} \cdot \operatorname{Pr}\left\{\operatorname{vol}\left(\mathcal{S}_{R}^{\prime}\right) \leq C\right\} \\
& +\operatorname{Pr}\left\{\mathbf{y} \in \mathcal{S}_{R}^{\prime} \mid \operatorname{vol}\left(\mathcal{S}_{R}^{\prime}\right)>C\right\} \cdot \operatorname{Pr}\left\{\operatorname{vol}\left(\mathcal{S}_{R}^{\prime}\right)>C\right\} \\
< & \operatorname{Pr}\left\{\mathbf{y} \in \mathcal{S}_{R}^{\prime} \mid \operatorname{vol}\left(\mathcal{S}_{R}^{\prime}\right) \leq C\right\}+\operatorname{Pr}\left\{\operatorname{vol}\left(\mathcal{S}_{R}^{\prime}\right)>C\right\} \quad(100)
\end{aligned}
$$

where $C$ is a positive number.
We then evaluate the two terms in (100) separately. We use the same settings as Lemmas 1 and 2, i.e., $P_{\mathrm{v}} \geq \rho^{2} / \Phi^{2 N_{\mathrm{B}} / N_{\mathrm{E}}}$, $\rho>\kappa(d)$. We set

$$
C=\pi^{N_{\mathrm{E}}} P_{\mathrm{v}}^{N_{\mathrm{E}}}\left(\frac{\rho}{\kappa(d)}\right)^{-N_{\mathrm{B}}} .
$$

1) $\operatorname{Pr}\left\{\mathbf{y} \in \mathcal{S}_{R}^{\prime} \mid \operatorname{vol}\left(\mathcal{S}_{R}^{\prime}\right) \leq C\right\}$ : Let $\mathcal{S}_{\mathrm{C}}$ be a sphere centered at $\mathbf{G V}_{1} \mathbf{u}$, where $\operatorname{vol}\left(\mathcal{S}_{\mathrm{C}}\right)=C$. Let $\mathcal{S}_{\mathrm{C} 0}$ be a sphere centered at the origin, where $\operatorname{vol}\left(\mathcal{S}_{\mathrm{C} 0}\right)=C$. Recalling that Alice knows $\mathbf{Z}$ and $\mathbf{v}_{0}$. For $\mathbf{G}$, Alice knows its statistics, but doesn't know its realization. Therefore, from Alice perspective, $\tilde{\mathbf{n}}_{\mathrm{v}}=\mathbf{G Z}_{\mathbf{v}_{0}}$ has i.i.d. $\mathcal{N}_{\mathbb{C}}\left(0, P_{\mathrm{v}}\right)$ components [30].

Therefore, we have

$$
\begin{aligned}
\operatorname{Pr} & \left\{\mathbf{y} \in \mathcal{S}_{R}^{\prime} \mid \operatorname{vol}\left(\mathcal{S}_{R}^{\prime}\right) \leq C\right\} \\
& \leq \operatorname{Pr}\left\{\mathbf{y} \in \mathcal{S}_{\mathrm{C}}\right\} \\
& =\int_{\mathcal{S}_{\mathrm{C} 0}} f\left(\tilde{\mathbf{n}}_{\mathrm{v}}\right) d \tilde{\mathbf{n}}_{\mathrm{v}} \\
& \leq \frac{C}{\pi^{N_{\mathrm{E}}} P_{\mathrm{v}}^{N_{\mathrm{E}}}} \\
& =(\rho / \kappa(d))^{-N_{\mathrm{B}}},
\end{aligned}
$$

where $f\left(\tilde{\mathbf{n}}_{\mathrm{v}}\right)$ is the probability density function (pdf) of $\tilde{\mathbf{n}}_{\mathrm{v}}$. The last inequality holds since

$$
\begin{aligned}
f\left(\tilde{\mathbf{n}}_{\mathrm{v}}\right) & =\frac{1}{\pi^{N_{\mathrm{E}}} P_{\mathrm{V}}^{N_{\mathrm{E}}}} \exp \left(-\frac{\left\|\tilde{\mathbf{n}}_{\mathrm{v}}\right\|^{2}}{\tilde{\sigma}_{\mathrm{v}}^{2}}\right) \\
& \leq \frac{1}{\pi^{N_{\mathrm{E}}} P_{\mathrm{v}}^{N_{\mathrm{E}}}} .
\end{aligned}
$$

2) $\operatorname{Pr}\left\{\operatorname{vol}\left(\mathcal{S}_{R}^{\prime}\right)>C\right\}$ : Since $\operatorname{vol}\left(\mathcal{S}_{R}^{\prime}\right)=d \cdot \operatorname{vol}\left(\Lambda_{\mathbb{C}}\right)$, we have $\operatorname{Pr}\left\{\operatorname{vol}\left(\mathcal{S}_{R}^{\prime}\right)>C\right\}=\operatorname{Pr}\left\{\Delta(d)>(\rho / \kappa(d))^{-N_{\mathrm{B}}}\right\}$.

From (100), (102), (104), and (39), as $N_{\mathrm{B}} \rightarrow \infty$,

$$
F_{D}\left(d, P_{\mathrm{v}}\right)<O\left(\left(\frac{\rho}{\kappa(d)}\right)^{-N_{\mathrm{B}}}\right) .
$$

From (100), (102), (104), and (41), when $N_{\mathrm{B}}$ is finite,

$$
F_{D}\left(d, P_{\mathrm{v}}\right)<\left(\frac{\rho}{\kappa(d)}\right)^{-N_{\mathrm{B}}}+\Upsilon\left(\frac{\rho}{\kappa(d)}\right) .
$$

\section{Proof of Theorem 2}

From (48) and (33), we have

$$
P_{\text {out }}(d)=F_{D}\left(d, P_{\mathrm{v}}\right)
$$

Let $\rho=\varepsilon^{-1 / N_{\min }} \kappa(d)$, for arbitrarily small $\varepsilon>0$. We have

$$
(\rho / \kappa(d))^{-N_{\mathrm{B}}}=\varepsilon^{N_{\mathrm{B}} / N_{\min }} \leq \varepsilon .
$$


From Lemma 3, (108), and (44), if $P_{\mathrm{v}} \geq \rho^{2} / \Phi^{2 N_{\mathrm{B}} / N_{\mathrm{E}}}$, we have

$$
F_{D}\left(d, P_{\mathrm{v}}\right)<\varepsilon+\Upsilon\left(\varepsilon^{-1 / N_{\min }}\right)=O(\varepsilon),
$$

or equivalently,

$$
P_{\text {out }}(d)<O(\varepsilon) \text {. }
$$

\section{E. Proof of Lemma 4}

Recalling that

$$
\begin{aligned}
R_{\max }\left(P_{\mathbf{v}}\right) & =\max _{\|\mathbf{v}\|^{2} \leq P_{\mathbf{v}}}\|\mathbf{G} \mathbf{Z v}\|, \\
r_{\text {eff }}\left(\Lambda_{\mathbb{C}}\right) & =\sqrt{N_{\mathrm{B}} /(\pi e)}\left|\operatorname{det}\left(\left(\mathbf{G V}_{1}\right)^{H}\left(\mathbf{G V}_{1}\right)\right)\right|^{\frac{1}{2 N_{\mathrm{B}}}} .
\end{aligned}
$$

From (29), applying Cauchy-Schwarz inequality,

$$
R_{\text {max }}^{2}\left(P_{\mathrm{v}}\right)=\lambda_{\max } P_{\mathrm{v}} \leq P_{\mathrm{v}}\|\mathbf{G} \mathbf{Z}\|_{F}^{2} .
$$

From Alice perspective, $\mathbf{G Z}$ is a complex Gaussian random matrix with i.i.d. components. Thus, $\|\mathbf{G Z Z}\|_{F}^{2}$ can be expressed in terms of a Chi-squared random variable:

$$
\|\mathbf{G Z Z}\|_{F}^{2}=\frac{1}{2} \mathcal{X}^{2}\left(2 N_{\mathrm{E}}\left(N_{\mathrm{A}}-N_{\mathrm{B}}\right)\right) .
$$

According to (82), $r_{\text {eff }}\left(\Lambda_{\mathbb{C}}\right)$ can be expressed in terms of $N_{\mathrm{B}}$ independent Chi-squared variables:

$r_{\text {eff }}\left(\Lambda_{\mathbb{C}}\right)=\sqrt{N_{\mathrm{B}} /(\pi e)}\left(\prod_{j=1}^{N_{\mathrm{B}}} \frac{1}{2} \mathcal{X}^{2}\left(2\left(N_{\mathrm{E}}-j+1\right)\right)\right)^{\frac{1}{2 N_{\mathrm{B}}}}$.

Moreover, since $\mathbf{G} \mathbf{V}_{1}$ and $\mathbf{G Z}$ are mutually independent [30], $R_{\max }\left(P_{\mathrm{v}}\right)$ and $r_{\text {eff }}\left(\Lambda_{\mathbb{C}}\right)$ are independent.

Then, we have

$$
\begin{aligned}
& \operatorname{Pr}\left\{\frac{2 R_{\max , i}\left(P_{\mathrm{v}}\right)}{\sqrt{M} r_{\mathrm{eff}, i}\left(\Lambda_{\mathbb{C}}\right)}<x\right\} \\
& \geq \operatorname{Pr}\left\{\frac{P_{\mathrm{v}}\|\mathbf{G} \mathbf{Z}\|_{F}^{2}}{r_{\mathrm{eff}}\left(\Lambda_{\mathbb{C}}\right)^{2}}<\frac{x^{2} M}{4}\right\} \\
& =\operatorname{Pr}\left\{\frac{\mathcal{X}^{2}\left(2 N_{\mathrm{E}}\left(N_{\mathrm{A}}-N_{\mathrm{B}}\right)\right)}{\left(\prod_{j=1}^{N_{\mathrm{B}}} \mathcal{X}^{2}\left(2\left(N_{\mathrm{E}}-j+1\right)\right)\right)^{\frac{1}{N_{\mathrm{B}}}}}<\frac{x^{2} M N_{\mathrm{B}}}{4 \pi e P_{\mathrm{v}}}\right\} \\
& \stackrel{a}{\geq} \operatorname{Pr}\left\{\frac{\mathcal{X}^{2}\left(2 N_{\mathrm{E}}\left(N_{\mathrm{A}}-N_{\mathrm{B}}\right)\right)}{\frac{N_{\mathrm{B}}}{\sum_{j=1}^{N_{\mathrm{B}}} \frac{1}{\mathcal{X}^{2}\left(2\left(N_{\mathrm{E}}-j+1\right)\right)}}}<\frac{x^{2} M N_{\mathrm{B}}}{4 \pi e P_{\mathrm{v}}}\right\} \\
& =\operatorname{Pr}\left\{\sum_{j=1}^{N_{\mathrm{B}}} \frac{\mathcal{X}^{2}\left(2 N_{\mathrm{E}}\left(N_{\mathrm{A}}-N_{\mathrm{B}}\right)\right)}{\mathcal{X}^{2}\left(2\left(N_{\mathrm{E}}-j+1\right)\right)}<\frac{x^{2} M N_{\mathrm{B}}^{2}}{4 \pi e P_{\mathrm{v}}}\right\} \\
& >\prod_{j=1}^{N_{\mathrm{B}}} \operatorname{Pr}\left\{\frac{\mathcal{X}^{2}\left(2 N_{\mathrm{E}}\left(N_{\mathrm{A}}-N_{\mathrm{B}}\right)\right)}{\mathcal{X}^{2}\left(2\left(N_{\mathrm{E}}-j+1\right)\right)} \leq \frac{x^{2} M N_{\mathrm{B}}}{4 \pi e P_{\mathrm{v}}}\right\} \\
& =\prod_{j=1}^{N_{\mathrm{B}}} \operatorname{Pr}\left\{\mathcal{F}\left(2 N_{\mathrm{E}}\left(N_{\mathrm{A}}-N_{\mathrm{B}}\right), 2\left(N_{\mathrm{E}}-j+1\right)\right) \leq g(x, j)\right\},
\end{aligned}
$$

where $g(x, j)$ is given in (71), and $\mathcal{F}\left(k_{1}, k_{2}\right)$ represents an $\mathcal{F}$-distributed random variable with $k_{1}$ and $k_{2}$ degrees of freedom. (a) holds due to the inequality of geometric and harmonic means. (b) holds by induction on the fact that if the non-negative random variables $A_{i}, 1 \leq i \leq N$, are mutually independent, given a constant $C>0$,

$\operatorname{Pr}\left\{\sum_{i=1}^{N} A_{i}<C\right\}>\operatorname{Pr}\left\{A_{1} \leq C / N ; \sum_{i=2}^{N} A_{i} \leq C(N-1) / N\right\}$

$$
=\operatorname{Pr}\left\{A_{1} \leq C / N\right\} \operatorname{Pr}\left\{\sum_{i=2}^{N} A_{i} \leq C(N-1) / N\right\} .
$$

Since the cdf of $\mathcal{F}\left(k_{1}, k_{2}\right)$ can be expressed using the regularized incomplete beta function [27], the final expression of (116) is given in (70).

\section{F. Proof of Theorem 3}

From Alice perspective, $L_{i}$ is a function of $\mathbf{G}_{i}$. Since $\left\{\mathbf{G}_{i}\right\}_{1}^{B}$ are mutually independent, $\left\{L_{i}\right\}_{1}^{B}$ are mutually independent. From (66), we have

$$
P_{\mathrm{F}, \text { out }}(d, B)=\prod_{i=1}^{B} \operatorname{Pr}\left\{L_{i}<d\right\} .
$$

We then evaluate $\operatorname{Pr}\left\{L_{i}<d\right\}$. For simplicity, we remove the index $i$. According to Theorem 2, with $P_{\mathrm{v}}=\varepsilon^{-2 / N_{\min }} \kappa(d)^{2} /$ $\Phi^{2 N_{\mathrm{B}} / N_{\mathrm{E}}}$, we have

$$
\operatorname{Pr}(D<d)<O(\varepsilon) .
$$

We can upper bound $\operatorname{Pr}\{L<d\}$ by

$$
\begin{aligned}
\operatorname{Pr}\{L<d\} \\
=\operatorname{Pr}\{L<d \mid D \geq d\} \operatorname{Pr}\{D \geq d\} \\
\quad+\operatorname{Pr}\{L<d \mid D<d\} \operatorname{Pr}\{D<d\} \\
\leq \operatorname{Pr}\{L<D \mid D \geq d\} \operatorname{Pr}\{D \geq d\}+O(\varepsilon) \\
\leq \operatorname{Pr}\{L<D\}+O(\varepsilon) .
\end{aligned}
$$

We then evaluate $\operatorname{Pr}\{L<D\}$.

$$
\begin{aligned}
\operatorname{Pr}\{L<D\}= & \operatorname{Pr}\left\{L<D \mid \Theta\left(P_{\mathrm{v}}\right)<\varepsilon\right\} \operatorname{Pr}\left\{\Theta\left(P_{\mathrm{v}}\right)<\varepsilon\right\} \\
& +\operatorname{Pr}\left\{L<D \mid \Theta\left(P_{\mathrm{v}}\right) \geq \varepsilon\right\} \operatorname{Pr}\left\{\Theta\left(P_{\mathrm{v}}\right) \geq \varepsilon\right\} \\
\leq & \operatorname{Pr}\left\{L<D \mid \Theta\left(P_{\mathrm{v}}\right)<\varepsilon\right\}+\operatorname{Pr}\left\{\Theta\left(P_{\mathrm{v}}\right) \geq \varepsilon\right\},
\end{aligned}
$$

where $\Theta\left(P_{\mathrm{v}}\right)$ is given in (69).

We then evaluate the two terms in (121), separately.

1) $\operatorname{Pr}\left\{L<D \mid \Theta\left(P_{\mathrm{v}}\right)<\varepsilon\right\}$ : Recalling that

$$
\begin{aligned}
\mathbf{y} & =\mathbf{G V}_{1} \mathbf{u}+\mathbf{G} \mathbf{Z} \mathbf{v}, \\
\Lambda_{\mathrm{F}} & =\left\{\mathbf{G V}_{1} \mathbf{u}, \mathbf{u} \in \tilde{\mathcal{Q}}^{N_{\mathrm{B}}}\right\} .
\end{aligned}
$$


Since $L=\left|S_{R_{\max }} \cap \Lambda_{\mathrm{F}}\right|$, we begin by checking the boundary of $\Lambda_{\mathrm{F}}$. Let $\mathbf{O}$ be the center point of $\Lambda_{\mathrm{F}}$. According to [33], for the Gaussian random lattice basis $\mathbf{G V}_{1}$, the boundary of $\Lambda_{\mathrm{F}}$ can be approximated by a sphere $S_{\mathrm{F}, \mathrm{S}}$ centered at $\mathbf{O}$ with radius $\sqrt{M} r_{\text {eff }}\left(\Lambda_{\mathbb{C}}\right)$, where $r_{\text {eff }}\left(\Lambda_{\mathbb{C}}\right)$ is given in (21).

Given $\Theta\left(P_{\mathrm{v}}\right)<\varepsilon$ and $\varepsilon<1$, we have $\sqrt{M} r_{\text {eff }}\left(\Lambda_{\mathbb{C}}\right)>$ $2 R_{\max }\left(P_{\mathrm{v}}\right)$. We define a concentric sphere $S_{\mathrm{F}, \mathrm{C}}$ with radius $\sqrt{M} r_{\text {eff }}\left(\Lambda_{\mathbb{C}}\right)-2 R_{\max }\left(P_{\mathrm{v}}\right)$, where $R_{\max }\left(P_{\mathrm{v}}\right)$ is given in (29). We then check when $L=D$ given $\Theta\left(P_{\mathrm{v}}\right)<\varepsilon$.

If $\mathbf{G V}_{1} \mathbf{u} \in S_{\mathrm{F}, \mathrm{C}}$, using triangle inequality, we have

$$
\begin{aligned}
\|\mathbf{y}-\mathbf{O}\| & \leq\left\|\mathbf{G V}_{1} \mathbf{u}-\mathbf{O}\right\|+\|\mathbf{G} \mathbf{Z} \mathbf{v}\| \\
& \leq \sqrt{M} r_{\mathrm{eff}}\left(\Lambda_{\mathbb{C}}\right)-R_{\max }\left(P_{\mathrm{v}}\right) .
\end{aligned}
$$

We then check the locations of the $D$ elements in $S_{R_{\max }} \cap$ $\Lambda_{\mathbb{C}}(56)$, denoted by, $\mathbf{G V}_{1} \mathbf{u}_{t}^{\prime}, 1 \leq t \leq D$. Note that

$$
\left\|\mathbf{G V}_{1} \mathbf{u}_{t}^{\prime}-\mathbf{y}\right\| \leq R_{\max }\left(P_{\mathrm{v}}\right) .
$$

From (124) and (125), using triangle inequality, for all $t$,

$\left\|\mathbf{G V}_{1} \mathbf{u}_{t}^{\prime}-\mathbf{O}\right\| \leq\|\mathbf{y}-\mathbf{O}\|+\left\|\mathbf{G V}_{1} \mathbf{u}_{t}^{\prime}-\mathbf{y}\right\| \leq \sqrt{M} r_{\mathrm{eff}}\left(\Lambda_{\mathbb{C}}\right)$.

Therefore, $S_{R_{\max }} \cap \Lambda_{\mathbb{C}} \subset \Lambda_{\mathrm{F}}$, i.e., $L=D$.

If $\mathbf{G V}_{1} \mathbf{u} \notin S_{\mathrm{F}, \mathrm{C}}$, there is a probability that $L<D$.

Therefore, we have

$\operatorname{Pr}\left\{L<D \mid \Theta\left(P_{\mathrm{v}}\right)<\varepsilon\right\}<\operatorname{Pr}\left\{\mathbf{G V}_{1} \mathbf{u} \notin S_{\mathrm{F}, \mathrm{C}}\right\}$.

Since $\mathbf{G V}_{1} \mathbf{u}$ is uniformly distributed over $S_{\mathrm{F}, \mathrm{S}}$, we have

$$
\begin{aligned}
\operatorname{Pr}\left\{\mathbf{G V}_{1} \mathbf{u} \in S_{\mathrm{F}, \mathrm{C}}\right\} & =\frac{\operatorname{vol}\left(S_{\mathrm{F}, \mathrm{C}}\right)}{\operatorname{vol}\left(S_{\mathrm{F}, \mathrm{S}}\right)} \\
& =\left(1-\Theta\left(P_{\mathrm{v}}\right)\right)^{2 N_{\mathrm{B}}}>(1-\varepsilon)^{2 N_{\mathrm{B}}} .
\end{aligned}
$$

Based on (127) and (128), we have

$\operatorname{Pr}\left\{L<D \mid \Theta\left(P_{\mathrm{v}}\right)<\varepsilon\right\}<1-(1-\varepsilon)^{2 N_{\mathrm{B}}}=O(\varepsilon)$.

2) $\operatorname{Pr}\left\{\Theta\left(P_{\mathrm{v}}\right) \geq \varepsilon\right\}$ : Using Lemma 4 with $M \geq \varepsilon^{-3-2 / N_{\min }} \kappa(d)^{2}$, we have

$$
\begin{aligned}
\operatorname{Pr}\left\{\Theta\left(P_{\mathrm{v}}\right)<\varepsilon\right\} & \geq \prod_{j=1}^{N_{\mathrm{B}}} B_{a, b(j)}\left(1-\frac{b(j)}{a g(\varepsilon, j)+b(j)}\right) \\
& \stackrel{a}{=} \prod_{j=1}^{N_{\mathrm{B}}} 1-B_{b(j), a}\left(\frac{b(j)}{a g(\varepsilon, j)+b(j)}\right) \\
& \stackrel{b}{=} \prod_{j=1}^{N_{\mathrm{B}}}\left(1-O\left(\varepsilon^{N_{\mathrm{E}}-j+1}\right)\right) \\
& >\left(1-O\left(\varepsilon^{N}\right)\right)^{N_{\mathrm{B}}},
\end{aligned}
$$

where $N=N_{\mathrm{E}}-N_{\mathrm{B}}+1$ and

$a=N_{\mathrm{E}}\left(N_{\mathrm{A}}-N_{\mathrm{B}}\right)$ and $b(j)=N_{\mathrm{E}}-j+1$. (a) and (b) hold due to the facts that

$$
\begin{aligned}
B_{a, b}(x) & =1-B_{b, a}(1-x), \\
B_{b(j), a}(x) & =O\left(x^{b(j)}\right), \text { for } x \rightarrow 0 .
\end{aligned}
$$

Consequently, we have

$$
\operatorname{Pr}\left\{\Theta\left(P_{\mathrm{v}}\right) \geq \varepsilon\right\}<1-\left(1-O\left(\varepsilon^{N}\right)\right)^{N_{\mathrm{B}}}=O\left(\varepsilon^{N}\right) .
$$

By substituting (121), (129), and (134) to (120), we have

$$
\operatorname{Pr}\{L<d\}<O(\varepsilon) .
$$

From (118) and (135), if $M \geq \varepsilon^{-3-2 / N_{\min }} \kappa(d)^{2}$ and $P_{\mathrm{v}}=\varepsilon^{-2 / N_{\min }} \kappa(d)^{2} / \Phi^{2 N_{\mathrm{B}} / N_{\mathrm{E}}}$, we have

$$
P_{\mathrm{F}, \text { out }}(d, B)<O\left(\varepsilon^{B}\right) \text {. }
$$

\section{ACKNOWLEDGMENT}

We thank Prof. T. Tao for his constructive comments and for suggesting references, and Prof. J. J. Boutros for his helpful discussions. We thank Prof. R. Zamir for pointing out the boundary effect of finite constellations.

\section{REFERENCES}

[1] C. E. Shannon, "Communication theory of secrecy systems," Bell Lab. Tech. J., vol. 28, no. 4, pp. 656-1715, Oct. 1949, Confidential Report.

[2] A. D. Wyner, "The wire-tap channel," Bell Syst. Tech. J., vol. 54, no. 8, pp. $1355-1387$, Oct. 1975

[3] I. Csiszár, "Almost independence and secrecy capacity," Problems Inf. Transmiss., vol. 32, no. 1, pp. 40-47, Jan./Mar. 1996.

[4] S. K. Leung-Yan-Cheong and M. E. Hellman, "The Gaussian wire-tap channel," IEEE Trans. Inf. Theory, vol. 24, no. 4, pp. 451-456, Jul. 1978.

[5] X. Tang, R. Liu, P. Spasojevic, and H. V. Poor, "The Gaussian wiretap channel with a helping interferer," in Proc. IEEE ISIT, Toronto, ON, Canada, Jul. 2008.

[6] P. K. Gopala, L. Lai, and H. E. Gamal, "On the secrecy capacity of fading channels," IEEE Trans. Inf. Theory, vol. 54, no. 10, pp. 4687-5403, Oct. 2008.

[7] T. Liu and S. Shamai, "A note on the secrecy capacity of the multiple antenna wiretap channel," IEEE Trans. Inf. Theory, vol. 55, no. 6, pp. 2547 2553, Jun. 2009.

[8] H. Mahdavifar and A. Vardy, "Achieving the secrecy capacity of wiretap channels using polar codes," IEEE Trans. Inf. Theory, vol. 57, no. 10, pp. 6428-6443, Oct. 2011.

[9] C. Ling, L. Luzzi, J.-C. Belfiore, and D. Stehlé, "Semantically secure lattice codes for the Gaussian wiretap channel," IEEE Trans. Inf. Theory, vol. 60 , no. 10 , pp. 6399-6416, Oct. 2014.

[10] C. W. Wong, T. F. Wong, and J. M. Shea, "Secret-sharing LDPC codes for the BPSK-constrained Gaussian wiretap channel," IEEE Trans. Inf. Forensics Security, vol. 6, no. 3, pp. 551-564, Sep. 2011.

[11] S. Goldwasser and S. Micali, "Probabilistic encryption," J. Comput. Syst. Sci., vol. 28, no. 2, pp. 270-299, Apr. 1984.

[12] W. Diffie and M. E. Hellman, "New directions in cryptography," IEEE Trans. Inf. Theory, vol. 22, no. 6, pp. 644-654, Nov. 1976.

[13] J. Hoffstein, J. Pipher, and J. H. Silverman, "NTRU: A ring based public key cryptosystem," in Proc. ANTS-III, vol. 1423, Lecture Notes in Computer Science, Jun. 1998, pp. 267-288.

[14] D. Micciancio, "Improving lattice based cryptosystems using the Hermite Normal Form," in Proc. CaLC, vol. 2146, Lecture Notes in Computer Science, J. Silverman, Ed., Mar. 29/30, 2001, pp. 126-145, Providence, RI, USA: Springer-Verlag.

[15] R. Ahlswede and I. Csiszár, "Common randomness in information theory and cryptography. I. secret sharing," IEEE Trans. Inf. Theory, vol. 39, no. 4, pp. 1121-1132, Jul. 1993.

[16] C. Bennett, G. Brassard, C. Crepeau, and U. Maurer, "Generalized privacy amplification," IEEE Trans. Inf. Theory, vol. 41, no. 6, pp. 1915-1923, Nov. 1995. 
[17] M. Bloch, J. Barros, M. Rodrigues, and S. McLaughlin, "Wireless information-theoretic security," IEEE Trans. Inf. Theory, vol. 54, no. 6, pp. 2515-2534, Jun. 2008.

[18] D. Abbasi-Moghadam, V. T. Vakili, and A. Falahati, "Combination of turbo coding and cryptography in Non-Geo satellite communication systems," in Proc. IST, Aug. 2008, pp. 666-670.

[19] S. Goel and R. Negi, "Guaranteeing secrecy using artificial noise," IEEE Trans. Wireless Commun., vol. 7, no. 6, pp. 2180-2189, Jun. 2008

[20] S. Fakoorian and A. L. Swindlehurst, "Solutions for the MIMO Gaussian wiretap channel with a cooperative jammer," IEEE Trans. Signal Process., vol. 59, no. 10, pp. 5013-5022, Oct. 2011.

[21] S. Liu, Y. Hong, and E. Viterbo, "Practical secrecy using artificial noise," IEEE Commun. Lett., vol. 17, no. 7, pp. 1483-1486, Jul. 2013.

[22] A. Khisti and G. W. Wornell, "Secure transmission with multiple antennas I: The MISOME wiretap channel," IEEE Trans. Inf. Theory, vol. 56, no. 7, pp. 3088-3104, Jul. 2010

[23] P. R. Geffe, "Secrecy systems approximating perfect and ideal secrecy," Proc. IEEE, vol. 53, no. 9, pp. 1229-1230, Sep. 1965.

[24] S. Liu, Y. Hong, and E. Viterbo, "On measures of information-theoretic security (invited paper)," to be presented at 2014 IEEE Information Theory Workshop (ITW'14), Hobart, Tasmania, Nov. 2-5, 2014.

[25] J. H. Conway and N. J. A. Sloane, Sphere Packings, Lattices, Groups, 2nd ed. New York, NY, USA: Springer-Verlag, 1993.

[26] R. Zamir, "Lattices are everywhere," in Proc. ITA Workshop, Feb. 2009, pp. 392-421.

[27] M. Abramowitz and I. Stegun, "Handbook of Mathematical Functions With Formulas, Graphs, Mathematical Tables," in Applied Mathematics, vol. 55. New York, NY, USA: Dover, 1955.

[28] X. Zhou and M. R. McKay, "Secure transmission with artificial noise over fading channels: Achievable rate and optimal power allocation," IEEE Trans. Veh. Technol., vol. 59, no. 8, pp. 3831-3842, Oct. 2010.

[29] X. Zhang, X. Zhou, and M. R. McKay, "Enhancing secrecy with multiantenna transmission in wireless Ad Hoc networks," IEEE Trans. Inf. Forensics Security, vol. 8, no. 11, pp. 1802-1814, Nov. 2013.

[30] E. Lukacs and E. P. King, "A property of the normal distribution," Ann. Math. Statist., vol. 25, no. 2, pp. 389-394, 1954.

[31] A. M. Tulino and S. Verdú, Random Matrix Theory and Wireless Communications. Delft, The Netherlands: Now Publishers, 2004.

[32] P. M. Lee, Bayesian Statistics: An Introduction, 4th ed. Hoboken, NJ, USA: Wiley, 2012.

[33] M. Ajtai, "Random lattices and a conjectured 0-1 law about their polynomial time computable properties," in Proc. IEEE Symposium FOCS, Vancouver, BC, Canada, Nov. 2002.

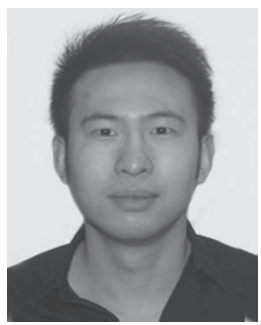

Shuiyin Liu (M'13) received the B.Eng. degree in electronic and information engineering from Beihang University, Beijing, China, in 2006 and the master's and Ph.D. degrees from Imperial College London, London, U.K., in 2007 and 2011 respectively. Since 2012, he has been working as a Research Fellow with the Department of Electrical and Computer Systems Engineering, Faculty of Engineering, Monash University, Melbourne, Australia. His current research interests include lattice coding for Gaussian and fading channels, cryptography, and

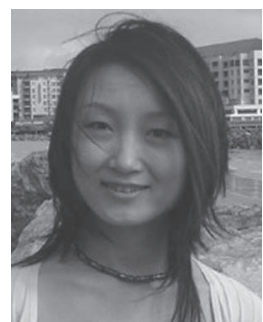

Yi Hong (S'00-M'05-SM'10) received the Ph.D. degree in electrical engineering and telecommunications from the University of New South Wales (UNSW), Sydney, Australia. She then worked with the Institute of Telecommunications Research, University of South Australia, Adelaide, Australia; with the Institute of Advanced Telecommunications, Swansea University, Swansea, U.K.; and with the University of Calabria, Cosenza, Italy. She is currently a Senior Lecturer with the Department of Electrical and Computer Systems Engineering, Faculty of Engineering, Monash University, Melbourne, Australia. Her research interests include information and communication theory with applications to telecommunication engineering. She is an Associate Editor for the European Transactions on Telecommunications. She is a General Cochair of the 2014 IEEE Information Theory Workshop, Hobart, Australia. She was the Publicity Chair at the 2009 IEEE Information Theory Workshop, Sicily, Italy. She is a Technical Program Committee Member for various IEEE conferences such as PIMRC and WCNC 2008, IEEE ICC 2011, and VTC 2011. During her Ph.D. studies, she was the recipient of an International Postgraduate Research Scholarship from the Commonwealth of Australia; a supplementary Engineering Award from the School of Electrical Engineering and Telecommunications, UNSW; and a Wireless Data Communication System Scholarship from UNSW. She was the recipient of the NICTA-ACoRN Earlier Career Researcher Award for a paper presented at the Australian Communication Theory Workshop (AUSCTW), Adelaide, Australia, in 2007.

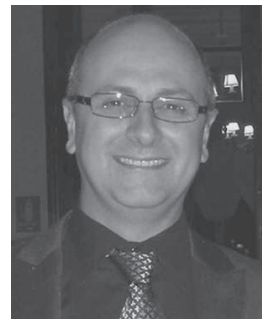

Emanuele Viterbo (M'95-SM'04-F'11) received the $\mathrm{Ph} . \mathrm{D}$. degree in electrical engineering from $\mathrm{Po}-$ litecnico di Torino, Torino, Italy, in 1995. He is currently a Professor with the Department of Electrical and Computer Systems Engineering, Faculty of Engineering, Monash University, Melbourne, Australia, where he is also an Associate Dean in Research Training. From 1990 to 1992, he was with the European Patent Office, The Hague, The Netherlands, as a Patent Examiner in the field of dynamic recording and error-control coding. Between 1995 and 1997, he held a postdoctoral position with Dipartimento di Elettronica, Politecnico di Torino, where he worked as an Assistant Professor and then as an Associate Professor from 1998 to 2005. From 1997 to 1998, he was a Postdoctoral Research Fellow with the Information Sciences Research Center, AT\&T Research, Florham Park, NJ, USA. From 2006 to 2009, he worked with the Department of Electronics, Computer Science and Systems (DEIS), University of Calabria, Cosenza, Italy, as a Full Professor. Since 1999, he has been an ISI Highly Cited Researcher. His main research interests include lattice codes for Gaussian and fading channels, algebraic coding theory, algebraic space-time coding, digital terrestrial television broadcasting, digital magnetic recording, and irregular sampling. He is an Associate Editor of the IEEE TRANSACTIONS ON INFORMATION THEORY, the European Transactions on Telecommunications, and the Journal of Communications and Networks, and he is a Guest Editor for the IEEE JOURNAL OF SELECTED TOPICS IN SIGNAL Processing: Special Issue Managing Complexity IN Multiuser MIMO SYSTEMS. He was the recipient of a NATO Advanced Fellowship from the Italian National Research Council in 1997. 